\title{
Incremental information of stock indicators
}

\author{
Dimitrios I. Vortelinos* \\ Lincoln Business School, University of Lincoln, LN6 7TS, UK
}

\section{A R T I C L E I N F O}

\section{Article history:}

Received 16 March 2015

Received in revised form 24 September 2015

Accepted 28 September 2015

Available online $\mathrm{xxxx}$

\section{Keywords:}

Stock market

Indicators

Asymmetry

HAR

\begin{abstract}
A B S T R A C T
The present paper is the first to examine the incremental information of stock indicators in the spot and futures stock markets. The properties of volatility series of indicators in relation to spot and futures stock indices are examined. Correlations between either the spot or futures stock indices and the corresponding indicators are examined for their properties. The asymmetry, heterogeneity and jump properties of volatilities and correlations are studied. Indicators offer information not captured in the corresponding futures and spot stock indices. Volatility and correlation in the stock market are accurately in-sample predicted via asymmetric and HAR models. The inclusion of indicators improves the in-sample modeling of volatility and correlation in the stock market.
\end{abstract}

(c) 2015 Elsevier Inc. All rights reserved.

\section{Introduction}

Financial indicators are useful for explaining the behavior of financial markets. In many times, they work as sentiment measures. These indicators can be thought of as the underlying market itself. The finance literature has not either analyzed or utilized these financial indicators. When, at the same time, they are very popular among professionals. This paper is one among very few papers to analyze the properties of seven major indicators of the US stock market. Their properties are compared to their underlying markets. As underlying markets, nine spot stock indices and five e-mini futures stock indices are used. The main research question of the paper is the incremental information of indicators, as depicted in the underlying spot and (e-mini) futures indices.

Incremental information of indicators is examined on: (a) distributional properties of returns, volatilities and correlations across indicators, spot stock indices and futures stock indices, (b) Granger-causality asymmetries on correlations between indicators and underlying (spot and futures) indices, and (c) the explanatory power of heterogeneity- and jumps-properties of indicators' volatility in correlations via a heterogeneity- and jumps-model (HAR-RV-J); and (d) the explanatory power of the continuous- and jumps-components of indicators' volatility in correlations via a continuous- and jumps-components model (HAR-RV-CJ).

Volatility is estimated using the realized (Parkinson) range estimator as in Martens and van Dijk (2007) and Todorova and Soucek (2014). Correlation series are estimated via the realized (Parkinson) range correlation estimator, as settled in Martens and van Dijk (2007). Jumps in volatility and correlation series are detected via the jump detection scheme introduced in Huang and Tauchen (2005) and finalized in Andersen, Bollerslev, and Diebold (2007).

In this paper, the distributional properties of returns, volatilities and correlations across indicators, spot stock indices and futures stock indices are examined via (a) the magnitude of the average-, standard deviation-, skewness- and kurtosis-values; (b) the CVM normality test; (c) the Ljung-Box serial correlation test in levels and squared series; and (d) comparisons of distributional properties (Kolmogorov-Smirnov test).

\footnotetext{
* Tel.: + 441522835634 .

E-mail address: dvortelinos@lincoln.ac.uk.
} 
Table 1

Realized ranges - Kolmogorov-Smirnov test.

\begin{tabular}{|c|c|c|c|c|c|c|c|}
\hline & TRIN & TICK & TIKI & ADV & $\mathrm{DECL}$ & UVOL & DVOL \\
\hline \multicolumn{8}{|c|}{ Panel A. Spot stock indices - indicators } \\
\hline INDU & $0.999^{*}$ & $1.000^{*}$ & $1.000^{*}$ & $0.403^{*}$ & $0.999^{*}$ & $0.998^{*}$ & $1.000^{*}$ \\
\hline p-Values & $<2.2 \mathrm{e}-16$ & $<2.2 \mathrm{e}-16$ & $<2.2 \mathrm{e}-16$ & $<2.2 \mathrm{e}-16$ & $<2.2 \mathrm{e}-16$ & $<2.2 \mathrm{e}-16$ & $<2.2 \mathrm{e}-16$ \\
\hline NDX & $0.992^{*}$ & $0.996^{*}$ & $1.000^{*}$ & $0.706^{*}$ & $1.000^{*}$ & $0.996^{*}$ & $1.000^{*}$ \\
\hline p-Values & $<2.2 \mathrm{e}-16$ & $<2.2 \mathrm{e}-16$ & $<2.2 \mathrm{e}-16$ & $<2.2 \mathrm{e}-16$ & $<2.2 \mathrm{e}-16$ & $<2.2 \mathrm{e}-16$ & $<2.2 \mathrm{e}-16$ \\
\hline INX & $0.992^{*}$ & $0.996^{*}$ & $1.000^{*}$ & $0.420^{*}$ & $0.996^{*}$ & $0.996^{*}$ & $1.000^{*}$ \\
\hline p-Values & $<2.2 \mathrm{e}-16$ & $<2.2 \mathrm{e}-16$ & $<2.2 \mathrm{e}-16$ & $<2.2 \mathrm{e}-16$ & $<2.2 \mathrm{e}-16$ & $<2.2 \mathrm{e}-16$ & $<2.2 \mathrm{e}-16$ \\
\hline COMPX & $0.991^{*}$ & $0.996^{*}$ & $1.000^{*}$ & $0.542^{*}$ & $1.000^{*}$ & 0.996* & $1.000^{*}$ \\
\hline p-Values & $<2.2 \mathrm{e}-16$ & $<2.2 \mathrm{e}-16$ & $<2.2 \mathrm{e}-16$ & $<2.2 \mathrm{e}-16$ & $<2.2 \mathrm{e}-16$ & $<2.2 \mathrm{e}-16$ & $<2.2 \mathrm{e}-16$ \\
\hline RUT & $0.990^{*}$ & $0.996^{*}$ & $1.000^{*}$ & $0.561^{*}$ & $1.000^{*}$ & $0.995^{*}$ & $1.000^{*}$ \\
\hline p-Values & $<2.2 \mathrm{e}-16$ & $<2.2 \mathrm{e}-16$ & $<2.2 \mathrm{e}-16$ & $<2.2 \mathrm{e}-16$ & $<2.2 \mathrm{e}-16$ & $<2.2 \mathrm{e}-16$ & $<2.2 \mathrm{e}-16$ \\
\hline OEX & $0.991^{*}$ & $0.996^{*}$ & $1.000^{*}$ & $0.418^{*}$ & $0.996^{*}$ & $0.996^{*}$ & $1.000^{*}$ \\
\hline p-Values & $<2.2 \mathrm{e}-16$ & $<2.2 \mathrm{e}-16$ & $<2.2 \mathrm{e}-16$ & $<2.2 \mathrm{e}-16$ & $<2.2 \mathrm{e}-16$ & $<2.2 \mathrm{e}-16$ & $<2.2 \mathrm{e}-16$ \\
\hline IDX & 0.993* & $0.997^{*}$ & $1.000^{*}$ & $0.477^{*}$ & $1.000^{*}$ & $0.996^{*}$ & $1.000^{*}$ \\
\hline p-Values & $<2.2 \mathrm{e}-16$ & $<2.2 \mathrm{e}-16$ & $<2.2 \mathrm{e}-16$ & $<2.2 \mathrm{e}-16$ & $<2.2 \mathrm{e}-16$ & $<2.2 \mathrm{e}-16$ & $<2.2 \mathrm{e}-16$ \\
\hline RUI & $0.992^{*}$ & $0.995^{*}$ & 0.999* & $0.403^{*}$ & $0.998^{*}$ & 0.997* & 0.999* \\
\hline p-Values & $<2.2 \mathrm{e}-16$ & $<2.2 \mathrm{e}-16$ & $<2.2 \mathrm{e}-16$ & $<2.2 \mathrm{e}-16$ & $<2.2 \mathrm{e}-16$ & $<2.2 \mathrm{e}-16$ & $<2.2 \mathrm{e}-16$ \\
\hline RUA & $0.991^{*}$ & $0.996^{*}$ & $0.998^{*}$ & $0.404^{*}$ & $1.000^{*}$ & $0.995^{*}$ & $0.998^{*}$ \\
\hline p-Values & $<2.2 \mathrm{e}-16$ & $<2.2 \mathrm{e}-16$ & $<2.2 \mathrm{e}-16$ & $<2.2 \mathrm{e}-16$ & $<2.2 \mathrm{e}-16$ & $<2.2 \mathrm{e}-16$ & $<2.2 \mathrm{e}-16$ \\
\hline \multicolumn{8}{|c|}{ Panel B. Futures stock indices - indicators } \\
\hline ES & $0.991^{*}$ & $0.995^{*}$ & $0.998^{*}$ & $0.479^{*}$ & $0.993^{*}$ & $0.995^{*}$ & $0.998^{*}$ \\
\hline p-Values & $<2.2 \mathrm{e}-16$ & $<2.2 \mathrm{e}-16$ & $<2.2 \mathrm{e}-16$ & $<2.2 \mathrm{e}-16$ & $<2.2 \mathrm{e}-16$ & $<2.2 \mathrm{e}-16$ & $<2.2 \mathrm{e}-16$ \\
\hline NQ & $0.991^{*}$ & $0.995^{*}$ & 0.999* & $0.653^{*}$ & $0.992^{*}$ & $0.996^{*}$ & $0.999 *$ \\
\hline p-Values & $<2.2 \mathrm{e}-16$ & $<2.2 \mathrm{e}-16$ & $<2.2 \mathrm{e}-16$ & $<2.2 \mathrm{e}-16$ & $<2.2 \mathrm{e}-16$ & $<2.2 \mathrm{e}-16$ & $<2.2 \mathrm{e}-16$ \\
\hline YM & 0992* & $0.996^{*}$ & $0.999 *$ & $0.444^{*}$ & $0.993^{*}$ & $0.996^{*}$ & $0.998^{*}$ \\
\hline p-Values & $<2.2 \mathrm{e}-16$ & $<2.2 \mathrm{e}-16$ & $<2.2 \mathrm{e}-16$ & $<2.2 \mathrm{e}-16$ & $<2.2 \mathrm{e}-16$ & $<2.2 \mathrm{e}-16$ & $<2.2 \mathrm{e}-16$ \\
\hline $\mathrm{TF}$ & $0.991^{*}$ & $0.996^{*}$ & 0.999* & $0.720^{*}$ & $0.998^{*}$ & $0.996^{*}$ & 0.999* \\
\hline p-Values & $<2.2 \mathrm{e}-16$ & $<2.2 \mathrm{e}-16$ & $<2.2 \mathrm{e}-16$ & $<2.2 \mathrm{e}-16$ & $<2.2 \mathrm{e}-16$ & $<2.2 \mathrm{e}-16$ & $<2.2 \mathrm{e}-16$ \\
\hline EMD & $0.992^{*}$ & $0.996^{*}$ & $1.000^{*}$ & $0.555^{*}$ & $1.000^{*}$ & 0.996* & $1.000^{*}$ \\
\hline p-Values & $<2.2 \mathrm{e}-16$ & $<2.2 \mathrm{e}-16$ & $<2.2 \mathrm{e}-16$ & $<2.2 \mathrm{e}-16$ & $<2.2 \mathrm{e}-16$ & $<2.2 \mathrm{e}-16$ & $<2.2 \mathrm{e}-16$ \\
\hline
\end{tabular}

Notes. Table 1. H0: the two estimators come from the same distribution. There are both statistic- and p-values of the Kolmogorov-Smirnov test. The distributional properties of realized range of spot stock indices compared to realized range of the corresponding stock indicators (panel A), and also compare the properties of realized range of futures stock indices to realized range of the corresponding stock indicators (panel B). * reveals significance in the $5 \%$ significance level.

Amira, Taamouti, and Tsafack (2011) analyzed the Granger-causality asymmetries on correlations are analyzed regarding returns- and volatilities-news. A recent study on asymmetries is Soucek and Todorova (2014). The present paper examines Granger-causality asymmetries on correlations between indicators and underlying (spot and futures) indices. The scope of asymmetric regressions is to detect, apart from asymmetries, the existence of incremental information from indicators.

The role of jumps in realized volatility was recently researched in Soucek and Todorova (2014). The benefit of modeling jumps in realized volatility is deployed in Liao (2013). A recent study utilizing the benefits of HAR modeling is Sevi (2013). Atak and Kapetanios (2013) compare the out-of-sample performance of HAR to factor models. In the present paper, the explanatory power of heterogeneity- and jumps-properties of indicators' volatility in correlations via a heterogeneity- and jumps-model (HAR-RV-J). The explanatory power of the continuous- and jumps-components of indicators' volatility in correlations via a continuous- and jumps-components model (HAR-RV-CJ).

The rest of the paper is organized as follows. Section 2 describes the data. Section 3 presents the realized volatility (correlation) estimation \& jumps detection. Section 4 presents a preliminary analysis of volatilities and correlations. Section 5 studies asymmetries. Section 6 examines heterogeneity and jumps. Section 7 summarizes the results.

\section{Data}

The present paper uses 5-min data, which are sampled further from 1-min data. The dataset includes seven stock indicators, ten underlying stock spot indices, and five underlying stock futures indices. The indicators ${ }^{1}$ used are: (i) NYSE Short Term Trade Index (symbol: TRIN), (ii) NYSE Issues Up/Down Ratio (TICK), (iii) DJIA Issues Up/Down Ratio (TIKI), (iv) NYSE Advancing Issues (ADV), (v) NYSE Declining Issues (DECL), (vi) NYSE Up Volume (UVOL), and (vii) NYSE Decline Volume (DVOL). The underlying stock spot indices are: (i) Dow Jones Industrial Average (INDU), (ii) Nasdaq 100 Index (NDX), (iii) S\&P 500 Index (INX), (iv) Nasdaq Composite Index (COMPX), (v) Russell 2000 Index (RUT), (vi) S\&P 100 Index (OEX), (vii) S\&P 400 Midcap Index (IDX), (viii) Russell 1000 Index (RUI), and (ix) Russell 3000 Index (RUA). The underlying stock futures indices are: (i) E-Mini S\&P 500 Continuous Contract (ES), (ii) E-Mini Nasdaq 100 Continuous Contract (NQ), (iii) Mini-sized Dow Futures Continuous Contract (YM), (iv) Mini Russell 2000 Continuous Contract (TF), and (v) E-Mini S\&P MidCap 400 Continuous Contract (EMD).

\footnotetext{
${ }^{1}$ For detailed professional analysis of Intraday indicators, see the Active Trader Magazine and markettells.com.
} 
Table 2

Realized ranges - Asymmetries.

\begin{tabular}{|c|c|c|c|}
\hline & $\beta$ & $\gamma$ & $R^{2}$ \\
\hline \multicolumn{4}{|c|}{ Panel A. Indicators } \\
\hline TRIN & $7.99 \mathrm{e}-6^{*}$ & $-7.38 \mathrm{e}-5^{*}$ & 0.006 \\
\hline TICK & $9.24 \mathrm{e}-7^{*}$ & $-7.67 e-6^{*}$ & $6.53 \mathrm{e}-4$ \\
\hline TIKI & $-1.84 \mathrm{e}-7^{*}$ & $2.37 e-6^{*}$ & $1.91 \mathrm{e}-4$ \\
\hline ADV & $-3.82 \mathrm{e}-11^{*}$ & $4.84 \mathrm{e}-10^{*}$ & $3.20 \mathrm{e}-4$ \\
\hline DECL & $5.05 e-8^{*}$ & $-3.94 \mathrm{e}-6^{*}$ & $4.50 \mathrm{e}-4$ \\
\hline UVOL & $4.88 \mathrm{e}-6^{*}$ & $-2.64 \mathrm{e}-5^{*}$ & $7.23 \mathrm{e}-4$ \\
\hline DVOL & $2.13 e-8^{*}$ & $-2.53 e-5^{*}$ & 0.001 \\
\hline \multicolumn{4}{|c|}{ Panel B. Spot stock indices } \\
\hline INDU & $5.84 \mathrm{e}-10^{*}$ & $-6.81 \mathrm{e}-9^{*}$ & 0.001 \\
\hline NDX & $2.60 \mathrm{e}-10^{*}$ & $-1.95 e-9 *$ & $1.15 \mathrm{e}-4$ \\
\hline INX & $6.55 \mathrm{e}-10^{*}$ & $-6.92 \mathrm{e}-9^{*}$ & 0.009 \\
\hline COMPX & $6.09 \mathrm{e}-10^{*}$ & $-3.80 \mathrm{e}-9^{*}$ & $5.34 \mathrm{e}-4$ \\
\hline RUT & $1.06 \mathrm{e}-9^{*}$ & $-7.31 \mathrm{e}-9^{*}$ & 0.004 \\
\hline OEX & $7.21 \mathrm{e}-10^{*}$ & $-7.42 \mathrm{e}-9^{*}$ & 0.006 \\
\hline IDX & $9.66 \mathrm{e}-10^{*}$ & $-6.37 e-9 *$ & 0.015 \\
\hline RUI & $6.78 \mathrm{e}-10^{*}$ & $-7.42 \mathrm{e}-9^{*}$ & 0.006 \\
\hline RUA & $8.80 \mathrm{e}-10^{*}$ & $-6.51 \mathrm{e}-9^{*}$ & 0.002 \\
\hline \multicolumn{4}{|c|}{ Panel C. Futures stock indices } \\
\hline ES & $1.26 \mathrm{e}-9^{*}$ & $4.70 \mathrm{e}-9^{*}$ & $8.64 \mathrm{e}-4$ \\
\hline NQ & $1.25 \mathrm{e}-9^{*}$ & $4.26 \mathrm{e}-9^{*}$ & $8.91 \mathrm{e}-4$ \\
\hline YM & $-1.47 e-9^{*}$ & $-1.36 \mathrm{e}-8^{*}$ & 0.019 \\
\hline $\mathrm{TF}$ & $-6.03 e-9^{*}$ & $-8.70 \mathrm{e}-9^{*}$ & 0.021 \\
\hline EMD & $-1.42 \mathrm{e}-10^{*}$ & $4.65 \mathrm{e}-10^{*}$ & $5.76 \mathrm{e}-4$ \\
\hline
\end{tabular}

Notes. Table 2 entries report the $R^{2}$ and the asymmetric coefficients from the asymmetric regression for volatilities (Eq. (9)). ${ }^{*}$ reveals significance in the $5 \%$ significance level.

The heteroscedasticity and autocorrelation consistent (Newey-West) standard errors used in the calculation of the corresponding significance levels; available upon request.

All data series begin on April 5, 2002 and end on October 14, 2011 with a total of 2400 trading days. All data are capped to $61 / 2$ trading hours per day; from 9:30 to $16: 00$ US Eastern time. ${ }^{2}$ So, the number of 5-min intraday prices per day is 78.

\subsection{Stock indicators}

Indicators of the US stock market are either unknown or poorly examined in the finance literature. This sub-section explains what the seven major indicators of the US stock market are. Moreover, there is some evidence of indicators literature. NYSE Short Term Trade Index (symbol: TRIN) is a measure of stock market strength that divides the advance/decline ratio by the advance volume/decline volume ratio. TRIN lower than 1 indicates volume is concentrated in declining issues; and, TRIN greater than 1 indicates volume is concentrated in advancing issues. NYSE Issues Up/Down Ratio (symbol: TICK), provided by data providers, is the number of stocks (out of 3700 NYSE stocks) whose current 1-min intraday price is higher than the previous (1-min) trade (uptrick) divided by (instead of minus, as usually) the number whose current 1-min intraday price is lower than the previous (1-min) trade (downtick). Because of the present analysis purposes, TICK is depicted as a percentage; so, it equals the provided number of stocks whose current 1-min intraday price is higher than the previous (1-min) trade (uptrick) divided by the number whose current 1-min intraday price is lower than the previous (1-min) trade (downtick) to the total 3700 NYSE stocks. DJIA Issues Up/Down Ratio (symbol: TIKI), provided by data providers, is represented the number of the Dow 30 Industrials that have traded lower in 1-min intraday price from the previous (1-min) trade subtracted from the number of 30 Industrials that traded higher in 1-min intraday price from the previous (1-min) trade (so, it ranged from +30 to -30 ). Because of the negative prices, TIKI indicator is changed for the purposes of the present analysis. I created TIKI as the ratio of the absolute value of the provided difference of the number of the Dow 30 Industrials that have traded higher in 1-min intraday price from the previous (1-min) trade to the number of 30 Industrials that traded lower in 1-min intraday price from the previous (1-min) trade to the total number of 30 for all 30 DJIA components. NYSE Advancing Issues (symbol: ADV) are the number of issues that traded (every 1-min) higher in 1-min intraday price from the previous 1-min close price. NYSE Declining Issues (symbol: DECL) are the number of issues that traded (every 1-min) lower in 1-min intraday price from the previous 1-min close price. NYSE Up Volume (symbol: UVOL) indicates the number of shares that have traded up (every 1-min) in 1-min intraday price from the previous day's close. NYSE Decline Volume (symbol: DVOL) indicates the number of shares that have traded down (every 1-min) in 1-min intraday price from the previous day's close.

\footnotetext{
2 The hours in which most US spot equity indices are traded. This trading period is known as American trading-time zone.
} 
Table 3

Realized range correlations - Mean.

\begin{tabular}{|c|c|c|c|c|c|c|c|}
\hline & TRIN & TICK & TIKI & ADV & DECL & UVOL & DVOL \\
\hline \multicolumn{8}{|c|}{ Panel A. Spot stock indices - indicators } \\
\hline INDU & -0.360 & 0.118 & 0.045 & 0.054 & -0.331 & 0.384 & -0.339 \\
\hline NDX & -0.291 & 0.101 & 0.039 & -0.331 & -0.310 & 0.335 & -0.310 \\
\hline INX & -0.316 & 0.098 & 0.032 & 0.042 & -0.342 & 0.370 & -0.330 \\
\hline СОМРX & -0.286 & 0.096 & 0.037 & -0.360 & -0.373 & 0.357 & -0.325 \\
\hline RUT & -0.137 & 0.024 & 0.019 & -0.032 & -0.369 & 0.310 & -0.278 \\
\hline OEX & -0.318 & 0.090 & 0.033 & -0.326 & -0.306 & 0.361 & -0.320 \\
\hline IDX & -0.173 & 0.055 & -0.032 & -0.029 & -0.340 & 0.316 & -0.275 \\
\hline RUI & -0.332 & 0.106 & 0.061 & -0.356 & -0.324 & 0.386 & -0.346 \\
\hline RUA & -0.328 & 0.114 & -0.034 & 0.034 & -0.3672 & 0.392 & -0.352 \\
\hline \multicolumn{8}{|c|}{ Panel B. Futures stock indices - indicators } \\
\hline ES & -0.288 & 0.113 & -0.017 & 0.313 & -0.292 & 0.303 & -0.286 \\
\hline NQ & -0.244 & 0.099 & -0.006 & -0.012 & -0.277 & 0.279 & -0.258 \\
\hline YM & -0.285 & 0.070 & 0.073 & -0.283 & -0.291 & 0.304 & -0.283 \\
\hline $\mathrm{TF}$ & -0.191 & 0.047 & -0.067 & -0.078 & -0.298 & 0.260 & -0.251 \\
\hline EMD & -0.137 & -0.019 & -0.082 & -0.251 & -0.273 & 0.210 & -0.202 \\
\hline
\end{tabular}

Notes. Table 3 reports the means of realized range correlation $\left(R C_{t}^{R R}\right)$ estimates of correlations between spot stock indices and the corresponding stock indicators (panel A); and also between futures stock indices and stock indicators (panel B).

\subsection{E-mini futures stock indices}

The present paper uses E-mini futures instead of the regular futures on US stock indices. Reason is the increasing popularity of these mini futures. Moreover, literature has poorly analyzed these contracts regardless their massive usage from financial analysts. The e-mini futures analyzed here are: ES (on S\&P 500), NQ (on Nasdaq 100), YM (on DJIA), TF (on Russell 2000), and EMD (S\&P MidCap). Recently, Chen, Fung, and Kao (2008) and Chung, Sheu, and Hsu (2010) examined its properties. Hasbrouck (2003) analyzed the ES, and NQ e-mini futures contracts. To the best of my knowledge, the present paper is the first researching the properties of the last three mini futures contracts.

\subsection{Spot stock indices}

The first nine heavily traded spot indices in the US stock market are analyzed: INDU, NDX, INX, COMPX, RUT, OEX, IDX, RUI, and RUA. Most of these spot stock indices have been examined in recent finance literature; see, Hendershott and Moulton (2011); Rosa (2011) and Chordia, Roll, and Subrahmanyman (2011).

\section{Realized volatility (correlation) estimation \& jumps detection}

\subsection{Realized volatility estimation}

Volatility is latent. However, the integrated volatility can be measured. Integrated Volatility is better estimated by quadratic variation. In its turn, quadratic variation can be estimated either parametrically or nonparametrically. In the present paper, volatility is nonparametrically estimated by a combined estimator between realized volatility and range. This estimator is entitled realized Parkinson range-based volatility estimator. Andersen, Bollerslev, Diebold and Labys (2001) theoretically settled and empirically examined the realized volatility estimator. Parkinson (1980) settled and introduced the range estimator in the

Table 4

Comparison of Realized range correlations - Kolmogorov-Smirnov test - Compare correlations between Futures stock indices and indicators to correlations between Spot stock indices and indicators.

\begin{tabular}{lllllll}
\hline & TRIN & TICK & TIKI & ADV & DECL & UVOL \\
\hline ES-INX & $0.152^{*}$ & $0.223^{*}$ & $0.526^{*}$ & $0.857^{*}$ & $0.190^{*}$ & $0.271^{*}$ \\
p-Value & $<2.2 \mathrm{e}-16$ & $<2.2 \mathrm{e}-16$ & $<2.2 \mathrm{e}-16$ & $<2.2 \mathrm{e}-16$ & $<2.2 \mathrm{e}-16$ & $0.212^{*}$ \\
NQ-NDX & $0.162^{*}$ & $0.166^{*}$ & $0.513^{*}$ & $0.905^{*}$ & $0.163^{*}$ & $0.201^{*}$ \\
p-Value & $<2.2 \mathrm{e}-16$ & $<2.2 \mathrm{e}-16$ & $<2.2 \mathrm{e}-16$ & $<2.2 \mathrm{e}-16$ & $<2.2 \mathrm{e}-16$ & $<2 \mathrm{e}-16$ \\
YM-INDU & $0.230^{*}$ & $0.189^{*}$ & $0.543^{*}$ & $0.924^{*}$ & $0.148^{*}$ & $0.195^{*}$ \\
p-Value & $<2.2 \mathrm{e}-16$ & $<2.2 \mathrm{e}-16$ & $<2.2 \mathrm{e}-16$ & $<2.2 \mathrm{e}-16$ & $<2.2 \mathrm{e}-16$ & $<4^{*}$ \\
TF-RUT & $0.162^{*}$ & $0.218^{*}$ & $0.523^{*}$ & $0.678^{*}$ & $0.223^{*}$ & $0.204^{*}$ \\
p-Value & $<2.2 \mathrm{e}-16$ & $<2.2 \mathrm{e}-16$ & $<2.2 \mathrm{e}-16$ & $<2.2 \mathrm{e}-16$ & $<2.2 \mathrm{e}-16$ & $0.188^{*}$ \\
EMD-IDX & $0.159^{*}$ & $0.245^{*}$ & $0.528^{*}$ & $0.741^{*}$ & $0.264^{*}$ & $<2.2 \mathrm{e}-16$ \\
p-Value & $<2.2 \mathrm{e}-16$ & $<2.2 \mathrm{e}-16$ & $<2.2 \mathrm{e}-16$ & $<2.2 \mathrm{e}-16$ & $<2.2 \mathrm{e}-16$ & $0.345^{*}$ \\
\hline
\end{tabular}

Notes. H0: the two estimators come from the same distribution. There are both statistic- and p-values of the Kolmogorov-Smirnov test between two correlations. * reveals significance in the $5 \%$ significance level. The alternative hypothesis is two-sized. For example, the statistic value for ES-INX and TRIN is the statistic value comparing the ES-TRIN realized correlation to the INX-TRIN realized correlation. 
Table 5

Realized range correlations - Asymmetric regression 1- $\beta$-coefficient $\& R^{2}$ (in parentheses).

\begin{tabular}{|c|c|c|c|c|c|c|c|}
\hline & TRIN & TICK & TIKI & ADV & DECL & UVOL & DVOL \\
\hline \multicolumn{8}{|c|}{ Panel A. Spot stock indices - indicators } \\
\hline INDU & $0.013 *(3.15 \mathrm{e}-3)$ & $0.057^{*}(3.35 \mathrm{e}-3)$ & $0.012 *(4.38 \mathrm{e}-3)$ & $0.056^{*}(1.42 \mathrm{e}-4)$ & $-0.023^{*}(2.26 \mathrm{e}-3)$ & $2.11 \mathrm{e}-3^{*}(6.71 \mathrm{e}-3)$ & $-7.84 \mathrm{e}-3^{*}(0.0007685)$ \\
\hline NDX & $-0.072^{*}(2.98 \mathrm{e}-3)$ & $6.78 \mathrm{e}-5^{*}(2.86 \mathrm{e}-8)$ & $0.011 *(1.49 \mathrm{e}-3)$ & $-0.381^{*}(5.56 \mathrm{e}-4)$ & $1.10 \mathrm{e}-3^{*}(7.04 \mathrm{e}-6)$ & $0.136 *(2.88 \mathrm{e}-3)$ & $2.24 \mathrm{e}-3^{*}(7.82 \mathrm{e}-5)$ \\
\hline INX & $-0.082^{*}(3.45 \mathrm{e}-3)$ & $4.51 \mathrm{e}-3^{*}(1.32 \mathrm{e}-4)$ & $6.49 \mathrm{e}-3^{*}(8.06 \mathrm{e}-4)$ & $-0.092 *(2.27 e-4)$ & $-0.036^{*}(5.08 \mathrm{e}-3)$ & $0.138 *(3.06 \mathrm{e}-3)$ & $-2.83 e-3^{*}(9.39 e-5)$ \\
\hline COMPX & $-0.074^{*}(3.08 \mathrm{e}-3)$ & $1.35 e-3^{*}(1.21 e-5)$ & $6.71 e-3^{*}(6.94 \mathrm{e}-4)$ & $-0.461^{*}(7.57 \mathrm{e}-4)$ & $-1.15 e-3^{*}(7.12 e-6)$ & $0.143^{*}(2.85 \mathrm{e}-3)$ & $1.21 \mathrm{e}-3^{*}(2.21 \mathrm{e}-5)$ \\
\hline RUT & $-0.049 *(2.89 \mathrm{e}-3)$ & $0.012 *(2.40 \mathrm{e}-3)$ & $-5.41 e-3^{*}(7.78 \mathrm{e}-4)$ & $-0.015^{*}(8.70 \mathrm{e}-6)$ & $-0.034^{*}(3.73 e-3)$ & $0.158 *(3.33 \mathrm{e}-3)$ & $2.22 \mathrm{e}-3^{*}(5.05 \mathrm{e}-5)$ \\
\hline OEX & $-0.086^{*}(3.43 e-3)$ & $3.56 e-3^{*}(8.96 e-5)$ & $8.19 \mathrm{e}-3^{*}(1.24 \mathrm{e}-3)$ & $-0.705^{*}(1.38 \mathrm{e}-3)$ & $-0.028^{*}(3.37 e-3)$ & $0.128 *(2.98 \mathrm{e}-3)$ & $-1.71 e-3^{*}(3.57 e-5)$ \\
\hline IDX & $-0.059 *(2.89 \mathrm{e}-3)$ & $8.78 \mathrm{e}-3 *(1.54 \mathrm{e}-3)$ & $7.80 \mathrm{e}-3^{*}(1.45 \mathrm{e}-3)$ & $0.036^{*}(4.48 \mathrm{e}-5)$ & $-0.045^{*}(5.79 \mathrm{e}-3)$ & $0.173 *(3.40 \mathrm{e}-3)$ & $-0.014^{*}(1.85 \mathrm{e}-3)$ \\
\hline RUI & $-0.092 *(3.51 e-3)$ & $-2.38 \mathrm{e}-3^{*}(3.35 \mathrm{e}-5)$ & $4.32 \mathrm{e}-3^{*}(3.42 \mathrm{e}-4)$ & $-0.949 *(2.25 \mathrm{e}-3)$ & $-0.015^{*}(7.91 \mathrm{e}-4)$ & $0.187 *(3.13 e-3)$ & $-1.98 \mathrm{e}-3^{*}(4.60 \mathrm{e}-5)$ \\
\hline RUA & $-0.087^{*}(3.44 \mathrm{e}-3)$ & $0.012^{*}(7.88 \mathrm{e}-4)$ & $0.010 *(2.06 \mathrm{e}-3)$ & $-0.127^{*}(4.23 \mathrm{e}-4)$ & $-0.044^{*}(6.99 \mathrm{e}-3)$ & $0.189 *(3.06 \mathrm{e}-3)$ & $-4.58 \mathrm{e}-3^{*}(2.46 \mathrm{e}-4)$ \\
\hline \multicolumn{8}{|c|}{ Panel B. Futures stock indices - indicators } \\
\hline ES & $-0.080^{*}(2.81 \mathrm{e}-3)$ & $0.015^{*}(1.13 \mathrm{e}-3)$ & $0.014^{*}(1.63 e-3)$ & $-3.58 \mathrm{e}-3^{*}(6.13 e-8)$ & $2.13 e-3^{*}(3.38 \mathrm{e}-5)$ & $0.090 *(2.64 \mathrm{e}-3)$ & $3.83 e-3^{*}(2.79 e-4)$ \\
\hline NQ & $-0.070 *(2.76 \mathrm{e}-3)$ & $0.013^{*}(1.02 \mathrm{e}-3)$ & $0.011 *(1.14 \mathrm{e}-3)$ & $-0.201^{*}(6.06 \mathrm{e}-4)$ & $8.62 e-4^{*}(5.19 e-6)$ & $0.086^{*}(2.68 \mathrm{e}-3)$ & $5.56 e-3^{*}(5.56 e-4)$ \\
\hline YM & $-0.076^{*}(2.88 \mathrm{e}-3)$ & $-0.015^{*}(1.14 \mathrm{e}-3)$ & $0.014^{*}(2.02 \mathrm{e}-3)$ & $-0.065^{*}(2.09 e-5)$ & $-2.80 \mathrm{e}-3^{*}(5.22 \mathrm{e}-5)$ & $0.091 *(2.74 \mathrm{e}-3)$ & $5.26 e-3^{*}(4.75 e-4)$ \\
\hline $\mathrm{TF}$ & $-0.053^{*}(2.79 \mathrm{e}-3)$ & $-0.011^{*}(7.40 \mathrm{e}-4)$ & $-2.03 e-3^{*}(5.43 e-5)$ & $0.125^{*}(2.85 e-4)$ & $8.84 \mathrm{e}-3^{*}(4.74 \mathrm{e}-4)$ & $0.069 *(8.20 \mathrm{e}-3)$ & $3.59 \mathrm{e}-3^{*}(2.22 \mathrm{e}-4)$ \\
\hline EMD & $-0.048^{*}(2.86 \mathrm{e}-3)$ & $1.37 e-3^{*}(1.29 \mathrm{e}-5)$ & $1.12 \mathrm{e}-3^{*}(1.66 \mathrm{e}-5)$ & $-0.169 *(1.02 \mathrm{e}-4)$ & $0.015^{*}(1.21 \mathrm{e}-3)$ & $0.055^{*}(2.58 \mathrm{e}-3)$ & $0.019 *(5.71 \mathrm{e}-3)$ \\
\hline
\end{tabular}

Notes. Table 5 reports the $R^{2}$ and $\beta$-coefficient (in parentheses) of the asymmetric regression 1 (Eq. (10)) for the realized range correlation ( $R C_{t}^{R R}$ ) estimates between spot stock indices and the corresponding stock indicators (panel A); and also between futures stock indices and stock indicators (panel B). * denotes significance in a $5 \%$ significance level.

The heteroscedasticity and autocorrelation consistent (Newey-West) standard errors used in the calculation of the corresponding significance levels; available upon request.

literature. With access to high-frequency data, Christensen, Oomen, and Podolskij (2010) and Christensen and Podolskij (2012) exploit the insights of realized volatility to construct more precise range-based estimators of Integrated Volatility. Recently, Todorova (2012) empirically examined the properties as well as the accuracy of the realized (Parkinson) range estimator. According to literature, this estimator is defined as:

$$
R V_{t}^{R R}=\frac{1}{4 \log (2)} \sum_{i=1}^{m}\left(h_{i, t}-l_{i, t}\right)^{2}
$$

where $h_{i, m}$ and $l_{i, m}$ are the within the $i$-th intraday interval ( $5 \mathrm{~min}$ ) high and low logarithmic prices for each $t$ trading day.

\subsection{Realized volatility jumps}

The jumps detection scheme mostly employed in the literature was introduced in Huang and Tauchen (2005) and finalized in Andersen et al. (2007). They suggest the bipower variation as the most accurate estimate of the integrated variance excluding the jumps:

$$
R V_{t}^{B P V}=\mu_{p}^{-2} \sum_{i=2}^{m}\left|r_{i, m}\right|\left|r_{i-1, m}\right|
$$

In general, $R V_{t}^{R R}-R V_{t}^{B P V} \rightarrow \lambda_{t}$ which is the jump component of volatility and $R V_{t}^{R R}$ denotes realized range. Huang and Tauchen (2005) proposed the following test statistic (also settled and extensively examined in Andersen et al., 2007):

$$
Z_{t}=m^{1 / 2} \frac{\log \left(R V_{t}^{R R} / R V_{t}^{B P V}\right)}{\left[\left(\mu_{1}^{-4}+2 \mu_{1}^{-2}-5\right)\left\{T P Q_{t}\left(R V_{t}^{B P V}\right)^{-2}\right\}\right]^{1 / 2}}
$$

A jump is indicated as $\widetilde{J}_{t}^{R V^{R R}}=\max \left(R V_{t}^{R R}-R V_{t}^{B P V}, 0\right)$. The following test-based version for defining a day with a significant jump is used:

$$
J_{t}^{R V^{R R}}=I\left(Z_{t}>\Phi_{\alpha}\right)\left(R V_{t}^{R R}-R V_{t}^{B P V}\right)
$$

The continuous component of volatility is defined as $C_{t}^{R V R R}=R V_{t}^{R R}-J_{t}^{R V R R}$ and $\Phi_{\alpha}$ is the critical value of the standard normal distribution at $\alpha$ level of significance. Here $J_{t}$ is the sample estimator of the theoretical jump component $\lambda_{t}$ in the sense that $J_{t} \rightarrow \lambda_{t}$. 
Realized range correlations - Asymmetric regression 2- $\beta$-coefficient \& $\gamma$-coefficient (in parentheses).

$$
\text { TRIN }
$$

TICK

TIKI

ADV

DECL

UVOL

DVOL

Panel A. Spot stock indices - indicator

INDU $\quad 1.16 \mathrm{e}-3^{*}\left(4.79 \mathrm{e}-4^{*}\right)$

NDX $7.86 \mathrm{e}-4^{*}\left(-7.81 \mathrm{e}-5^{*}\right)$

$7.81 \mathrm{e}-4^{*}\left(-3.09 \mathrm{e}-4^{*}\right)$

ADV

\section{$5.02 \mathrm{e}-3^{*}\left(7.33 \mathrm{e}-3^{*}\right)$}

$-0.119^{*}\left(0.049^{*}\right)$

年 $1.073-3^{*}(-)$

$2.89 \mathrm{e}-4^{*}\left(-1.36 \mathrm{e}-3^{*}\right) \quad 5.42 \mathrm{e}-4^{*}\left(1.43 \mathrm{e}-4^{*}\right)$

$6.49 \mathrm{e}-4^{*}(-)$

$-2.35 \mathrm{e}-3^{*}\left(-0.018^{*}\right)$

$-2.54 \mathrm{e}-4^{*}\left(-7.13 \mathrm{e}-3^{*}\right)$

$1.92 \mathrm{e}-3^{*}\left(-4.11 \mathrm{e}-3^{*}\right)$

$6.16 \mathrm{e}-4^{*}(-9.19 \mathrm{e}-4)$

$6.71 \mathrm{e}-4^{*}(-)$

$-0.136^{*}\left(0.048^{*}\right)$

$-2.08 \mathrm{e}-3^{*}\left(-7.28 \mathrm{e}-3^{*}\right)$

$-5.41 \mathrm{e}-4^{*}(-)$

$-0.018^{*}\left(0.016^{*}\right)$

$\begin{array}{ll}\text { RUT } & 5.66 \mathrm{e}-4^{*}\left(-2.89 \mathrm{e}-4^{*}\right) \\ \text { OEX } & -7.35 \mathrm{e}-5^{*}\left(-1.78 \mathrm{e}-3^{*}\right)\end{array}$

IDX $\quad 2.97 \mathrm{e}-4^{*}\left(-5.06 \mathrm{e}-4^{*}\right)$

$1.70 \mathrm{e}-3^{*}\left(-1.54 \mathrm{e}-4^{*}\right)$

$5.87 \mathrm{e}-4^{*}\left(-1.70 \mathrm{e}-4^{*}\right)$

$8.19 \mathrm{e}-4^{*}(-)$

$-0.135^{*}\left(-4.17 \mathrm{e}-3^{*}\right)$

.20e-3* $\left(3.29 \mathrm{e}-4^{*}\right)$

$7.80 \mathrm{e}-4^{*}(-)$

$0.010^{*}\left(-0.012^{*}\right)$

$1.51 \mathrm{e}-3^{*}\left(-3.90 \mathrm{e}-3^{*}\right)$

$1.27 \mathrm{e}-3^{*}\left(-7.79 \mathrm{e}-4^{*}\right)$

$1.61 \mathrm{e}-4^{*}\left(-1.04 \mathrm{e}-3^{*}\right)$

$4.32 \mathrm{e}-4^{*}(-)$

$-0.076^{*}\left(-0.137^{*}\right)$

$-2.17 \mathrm{e}-3^{*}\left(-6.24 \mathrm{e}-3^{*}\right)$

RUA $1.40 \mathrm{e}-3^{*}\left(-5.78 \mathrm{e}-4^{*}\right)$

$1.54 \mathrm{e}-3^{*}\left(3.70 \mathrm{e}-4^{*}\right)$

$1.04 \mathrm{e}-3^{*}(-)$

$-1.18 \mathrm{e}-3^{*}\left(-6.72 \mathrm{e}-3^{*}\right)$

$-3.14 \mathrm{e}-3^{*}\left(-7.76 \mathrm{e}-3^{*}\right)$

$1.82 \mathrm{e}-4^{*}\left(-5.26 \mathrm{e}-3^{*}\right)$

$-2.86 \mathrm{e}-3^{*}\left(-7.86 \mathrm{e}-3^{*}\right)$

Panel B. Futures stock indices - indicators

ES $\quad 4.37 \mathrm{e}-4^{*}\left(-4.94 \mathrm{e}-4^{*}\right)$

NQ $\quad 6.35 \mathrm{e}-5^{*}\left(-5.32 \mathrm{e}-4^{*}\right)$

$6.63 \mathrm{e}-4^{*}\left(-8.44 \mathrm{e}-4^{*}\right)$

$24 \mathrm{e}-3^{*}\left(-2.95 \mathrm{e}-4^{*}\right)$

$1.36 \mathrm{e}-3^{*}(-)$

$-3(-0.026)$

$1.87 \mathrm{e}-3^{*}\left(-3.64 \mathrm{e}-3^{*}\right)$

- $1.06 \mathrm{e}-3^{*}(-)$

$0.025^{*}\left(-0.035^{*}\right)$

$1.45 \mathrm{e}-3^{*}(-)$

EMD $\quad 7.62 \mathrm{e}-4^{*}\left(-4.92 \mathrm{e}-4^{*}\right)$

$4.88 e^{*}(-9.19-4)$

$1.12 \mathrm{e}-4^{*}(-)$

$-0.018^{*}\left(-0.015^{*}\right)$

$1.98 \mathrm{e}-3^{*}\left(-4.33 \mathrm{e}-3^{*}\right)$

$1.53 \mathrm{e}-3^{*}\left(-4.50 \mathrm{e}-3^{*}\right)$

$\left(0.010^{*}\right)$

$2.63 \mathrm{e}-3^{*}\left(-3.18 \mathrm{e}-3^{*}\right)$

$3.42 \mathrm{e}-3^{*}\left(-2.95 \mathrm{e}-3^{*}\right)$

$1.00 \mathrm{e}-3^{*}\left(1.13 \mathrm{e}-3^{*}\right)$

$2.90 \mathrm{e}-5^{*}\left(4.16 \mathrm{e}-4^{*}\right)$

$1.59 \mathrm{e}-3^{*}\left(1.93 \mathrm{e}-3^{*}\right)$

$5.08 \mathrm{e}-4^{*}\left(6.26 \mathrm{e}-4^{*}\right)$

$2.50 \mathrm{e}-3^{*}\left(1.49 \mathrm{e}-3^{*}\right)$

$8.50 \mathrm{e}-4^{*}\left(1.56 \mathrm{e}-3^{*}\right)$

$2.44 \mathrm{e}-3^{*}\left(2.79 \mathrm{e}-3^{*}\right)$

$1.30 \mathrm{e}-5^{*}\left(2.30 \mathrm{e}-3^{*}\right)$

$1.66 \mathrm{e}-3^{*}\left(1.27 \mathrm{e}-3^{*}\right)$

(E) Table 6 reports the $\beta$ - and $\gamma$-coefficient (the latter in parentheses) of the asymmetric regression 2 (Eq. (11)) for the realized corre

indicators (panel A); and also between futures stock indices and stock indicators (panel B). ${ }^{*}$ denotes significance in a $5 \%$ significance level.

The heteroscedasticity and autocorrelation consistent (Newey-West) standard errors used in the calculation of the corresponding significance levels; available upon request.

$-1.74 \mathrm{e}-4^{*}\left(-1.65 \mathrm{e}-3^{*}\right)$

(1.52e-4* $\left(-9.00 \mathrm{e}-4^{*}\right)$

$3.07 \mathrm{e}-4^{*}\left(1.02 \mathrm{e}-4^{*}\right)$

$3.94 \mathrm{e}-4^{*}\left(-9.74 \mathrm{e}-4^{*}\right)$

$2.68 \mathrm{e}-4^{*}\left(-3.74 \mathrm{e}-3^{*}\right)$

$3.23 \mathrm{e}-4^{*}\left(-9.39 \mathrm{e}-4^{*}\right)$

$-2.25 \mathrm{e}-4^{*}\left(-7.90 \mathrm{e}-4^{*}\right)$

$-3.50 \mathrm{e}-4^{*}\left(1.67 \mathrm{e}-4^{*}\right)$

$1.00 \mathrm{e}-3^{*}\left(-5.01 \mathrm{e}-4^{*}\right)$

$1.06 \mathrm{e}-3^{*}\left(-1.63 \mathrm{e}-4^{*}\right)$

$1.15 \mathrm{e}-3^{*}(-7.69$ $2.45 \mathrm{e}-3^{*}\left(1.14 \mathrm{e}-3^{*}\right)$ 
Table 7

Realized range correlations - Asymmetric regression 3- $\beta$ - \& $\gamma$-coefficient (in parentheses).

\begin{tabular}{|c|c|c|c|c|c|c|c|}
\hline & TRIN & TICK & TIKI & ADV & DECL & UVOL & DVOL \\
\hline \multicolumn{8}{|c|}{ Panel A. Spot stock indices - indicators } \\
\hline INDU & $-1.42 \mathrm{e}-3^{*}\left(1.19 \mathrm{e}-3^{*}\right)$ & $-2.76 e-6^{*}\left(1.12 e-5^{*}\right)$ & $3.88 \mathrm{e}-4^{*}(-)$ & $0.011^{*}\left(-6.83 \mathrm{e}-3^{*}\right)$ & $-3.93 e-3^{*}\left(6.87 e-3^{*}\right)$ & $0.0011334^{*}\left(-3.10 \mathrm{e}-3^{*}\right)$ & $-9.40 \mathrm{e}-4^{*}\left(1.83 \mathrm{e}-3^{*}\right)$ \\
\hline NDX & $-2.36 \mathrm{e}-3^{*}\left(1.02 \mathrm{e}-3^{*}\right)$ & $-9.15 e-7^{*}\left(1.24 e-5^{*}\right)$ & $-2.34 \mathrm{e}-3^{*}(-)$ & $-8.82 \mathrm{e}-3^{*}\left(0.045^{*}\right)$ & $-4.07 e-3^{*}\left(4.57 e-3^{*}\right)$ & $1.70 \mathrm{e}-3^{*}\left(-1.90 \mathrm{e}-3^{*}\right)$ & $-5.97 e-4^{*}\left(1.94 e-3^{*}\right)$ \\
\hline INX & $-1.90 \mathrm{e}-3^{*}\left(1.217 \mathrm{e}-3^{*}\right)$ & $8.05 e-6^{*}\left(5.09 e-6^{*}\right)$ & $-1.21 \mathrm{e}-3^{*}(-)$ & $-0.084 *(0.015 *)$ & $-3.41 \mathrm{e}-3^{*}\left(7.45 \mathrm{e}-3^{*}\right)$ & $1.70 \mathrm{e}-3^{*}\left(-3.47 \mathrm{e}-3^{*}\right)$ & $-6.16 e-4^{*}\left(1.85 e-3^{*}\right)$ \\
\hline COMPX & $-2.42 \mathrm{e}-3^{*}\left(1.04 \mathrm{e}-3^{*}\right)$ & $2.65 e-6^{*}\left(2.14 e-6^{*}\right)$ & $-1.12 \mathrm{e}-3^{*}(-)$ & $-0.028^{*}\left(0.062^{*}\right)$ & $-4.08 \mathrm{e}-3^{*}\left(4.99 e-3^{*}\right)$ & $1.73 e-3^{*}\left(-2.34 e-3^{*}\right)$ & $-6.77 \mathrm{e}-4^{*}\left(2.03 \mathrm{e}-3^{*}\right)$ \\
\hline RUT & $-1.43 e-3^{*}\left(9.82 e-4^{*}\right)$ & $1.67 e-6^{*}\left(-4.49 e-6^{*}\right)$ & $-5.20 \mathrm{e}-4^{*}(-)$ & $9.21 \mathrm{e}-3^{*}\left(0.060^{*}\right)$ & $-3.89 e-3^{*}\left(7.53 e-3^{*}\right)$ & $2.04 \mathrm{e}-3^{*}\left(-4.48 \mathrm{e}-3^{*}\right)$ & $-5.93 \mathrm{e}-4^{*}\left(1.92 \mathrm{e}-3^{*}\right)$ \\
\hline OEX & $-2.12 \mathrm{e}-3^{*}\left(1.52 \mathrm{e}-3^{*}\right)$ & $1.33 e-5^{*}\left(1.02 e-5^{*}\right)$ & $2.33 e-3^{*}(-)$ & $-0.044^{*}\left(0.218^{*}\right)$ & $-3.84 \mathrm{e}-3^{*}\left(7.19 \mathrm{e}-3^{*}\right)$ & $1.34 \mathrm{e}-3^{*}\left(-3.14 \mathrm{e}-3^{*}\right)$ & $-4.46 e-4^{*}\left(2.75 e-3^{*}\right)$ \\
\hline IDX & $-1.45 \mathrm{e}-3^{*}\left(7.30 \mathrm{e}-4^{*}\right)$ & $-6.11 e-7^{*}\left(-4.90 e-6^{*}\right)$ & $-1.09 \mathrm{e}-3^{*}(-)$ & $-0.034^{*}\left(0.033^{*}\right)$ & $-4.63 e-3^{*}\left(9.04 e-3^{*}\right)$ & $2.33 e-3^{*}\left(-4.43 e-3^{*}\right)$ & $-2.44 e-3^{*}\left(4.07 e-3^{*}\right)$ \\
\hline RUI & $-1.16 \mathrm{e}-3^{*}\left(-1.26 \mathrm{e}-4^{*}\right)$ & $-3.36 \mathrm{e}-5^{*}\left(1.83 \mathrm{e}-5^{*}\right)$ & $-2.33 \mathrm{e}-3^{*}(-)$ & $-0.080^{*}\left(0.172^{*}\right)$ & $-2.39 \mathrm{e}-3^{*}\left(4.66 \mathrm{e}-3^{*}\right)$ & $7.24 \mathrm{e}-4^{*}\left(-2.95 \mathrm{e}-3^{*}\right)$ & $-1.62 e-3^{*}\left(1.48 \mathrm{e}-3^{*}\right)$ \\
\hline RUA & $-1.43 e-3^{*}\left(6.10 e-4^{*}\right)$ & $-1.15 e-5^{*}\left(-4.72 e-6^{*}\right)$ & $2.89 \mathrm{e}-4^{*}(-)$ & $-0.053^{*}\left(0.122^{*}\right)$ & $-3.91 e-3^{*}\left(7.76 e-3^{*}\right)$ & $1.95 e-3^{*}\left(-3.53 e-3^{*}\right)$ & $-1.15 \mathrm{e}-4^{*}\left(1.78 \mathrm{e}-3^{*}\right)$ \\
\hline \multicolumn{8}{|c|}{ Panel B. Futures stock indices - indicators } \\
\hline ES & $-2.29 \mathrm{e}-3^{*}\left(1.52 \mathrm{e}-3^{*}\right)$ & $-1.38 e-5^{*}\left(3.77 e-7^{*}\right)$ & $5.40 \mathrm{e}-3^{*}(-)$ & $0.022^{*}\left(0.028^{*}\right)$ & $-3.98 \mathrm{e}-3^{*}\left(3.93 \mathrm{e}-3^{*}\right)$ & $1.17 \mathrm{e}-3^{*}\left(-1.87 \mathrm{e}-3^{*}\right)$ & $-7.90 \mathrm{e}-4^{*}\left(1.66 \mathrm{e}-3^{*}\right)$ \\
\hline $\mathrm{NQ}$ & $-2.18 \mathrm{e}-3^{*}\left(1.32 \mathrm{e}-3^{*}\right)$ & $2.68 \mathrm{e}-5^{*}\left(8.79 \mathrm{e}-6^{*}\right)$ & $4.20 \mathrm{e}-3^{*}(-)$ & $-0.090^{*}\left(0.118^{*}\right)$ & $-4.25 e-3^{*}\left(4.43 e-3^{*}\right)$ & $1.43 \mathrm{e}-3^{*}\left(-1.72 \mathrm{e}-3^{*}\right)$ & $-9.67 e-4^{*}\left(1.77 e-3^{*}\right)$ \\
\hline YM & $-1.01 \mathrm{e}-3^{*}\left(7.40 \mathrm{e}-4^{*}\right)$ & $-5.43 e-6^{*}\left(-2.05 e-6^{*}\right)$ & $-1.54 \mathrm{e}-3^{*}(-)$ & $-0.073^{*}\left(-0.016^{*}\right)$ & $-1.75 e-3^{*}\left(1.68 e-3^{*}\right)$ & $1.50 \mathrm{e}-3^{*}\left(-2.10 \mathrm{e}-3^{*}\right)$ & $-2.07 e-3^{*}\left(1.82 e-3^{*}\right)$ \\
\hline $\mathrm{TF}$ & $-1.09 \mathrm{e}-3^{*}\left(3.35 \mathrm{e}-4^{*}\right)$ & $5.66 \mathrm{e}-6^{*}\left(-1.46 \mathrm{e}-5^{*}\right)$ & $-4.12 \mathrm{e}-5^{*}(-)$ & $-1.19 \mathrm{e}-4^{*}\left(0.027^{*}\right)$ & $-3.13 e-3^{*}\left(3.14 e-3^{*}\right)$ & $8.91 e-4^{*}\left(-8.73 e-4^{*}\right)$ & $-1.17 \mathrm{e}-3^{*}\left(1.58 \mathrm{e}-3^{*}\right)$ \\
\hline EMD & $-2.77 e-4^{*}\left(3.90 e-4^{*}\right)$ & $-1.33 e-5^{*}\left(1.42 e-5^{*}\right)$ & $1.26 \mathrm{e}-3^{*}(-)$ & $-0.016^{*}\left(0.017^{*}\right)$ & $-4.13 e-3^{*}\left(4.64 e-3^{*}\right)$ & $1.63 e-3^{*}\left(-1.40 e-3^{*}\right)$ & $-1.94 \mathrm{e}-3^{*}\left(1.18 \mathrm{e}-3^{*}\right)$ \\
\hline
\end{tabular}

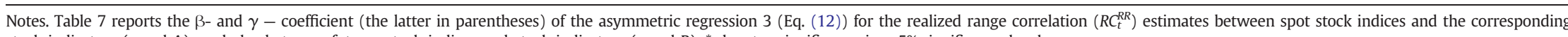
stock indicators (panel A); and also between futures stock indices and stock indicators (panel B). * denotes significance in a $5 \%$ significance level.

The heteroscedasticity and autocorrelation consistent (Newey-West) standard errors used in the calculation of the corresponding significance levels; available upon request. 
Table 8

Realized range correlations - Asymmetric regression 4- $\beta$ - \& $\gamma$-coefficient (in parentheses).

\begin{tabular}{lll}
\hline TRIN & TICK & TIKI
\end{tabular}

TIKI

ADV

DECL

UVOL

DVOL

Panel A. Spot stock indices - indicators

INDU $-1.42 \mathrm{e}-3^{*}\left(1.19 \mathrm{e}-3^{*}\right)$

NDX $\quad-2.37 \mathrm{e}-3^{*}\left(1.01 \mathrm{e}-3^{*}\right)$

INX $\quad-1.90 \mathrm{e}-3^{*}\left(1.21 \mathrm{e}-3^{*}\right)$

$-3.29 \mathrm{e}-6^{*}\left(1.10 \mathrm{e}-5^{*}\right)$

$-1.35 \mathrm{e}-7^{*}\left(1.21 \mathrm{e}-5^{*}\right)$

$3.88 \mathrm{e}-4^{*}(-)$

$\left(1.90 \mathrm{e}-3^{*}\left(1.21 \mathrm{e}-3^{*}\right)\right.$

$-2.34 \mathrm{e}-3^{*}(-)$

$-1.24 \mathrm{e}-3^{*}(-)$

$-1.12 \mathrm{e}-3^{*}(-)$

$-1.44 \mathrm{e}-3^{*}\left(9.75 \mathrm{e}-4^{*}\right)$

$2.33 \mathrm{e}-3^{*}(-)$

$-1.36 \mathrm{e}-5^{*}\left(1.01 \mathrm{e}-5^{*}\right)$

$-1.46 \mathrm{e}-3^{*}\left(7.24 \mathrm{e}-4^{*}\right)$

$-6.92 \mathrm{e}-7^{*}\left(4.88 \mathrm{e}-6^{*}\right)$

$-1.09 \mathrm{e}-3^{*}(-)$

$-2.33 \mathrm{e}-3^{*}(-)$

$-1.44 \mathrm{e}-3^{*}\left(6.04 \mathrm{e}-4^{*}\right)$

$-1.16 \mathrm{e}-5^{*}\left(-4.64 \mathrm{e}-6^{*}\right)$

$2.89 \mathrm{e}-4^{*}(-)$

$1.41 \mathrm{e}-5^{*}\left(2.17 \mathrm{e}-7^{*}\right)$

$-2.68 \mathrm{e}-5^{*}\left(8.75 \mathrm{e}-6^{*}\right)$
$-5.81 \mathrm{e}-6^{*}\left(2.23 \mathrm{e}-6^{*}\right)$

$5.40 \mathrm{e}-3^{*}(-)$

2.30e-3* $3^{*}\left(1.51 \mathrm{e}-3^{*}\right)$

4.20e- $3^{*}(-)$

$-1.01 \mathrm{e}-3^{*}\left(7.31 \mathrm{e}-4^{*}\right)$

$5.48 \mathrm{e}-6 *\left(-1.45 \mathrm{e}-5^{*}\right)$

$-1.54 \mathrm{e}-3^{*}(-)$

$-1.10 \mathrm{e}-3^{*}\left(3.32 \mathrm{e}-4^{*}\right)$

$-1.36 \mathrm{e}-5^{*}\left(1.41 \mathrm{e}-5^{*}\right)$

$-4.12 \mathrm{e}-5^{*}(-)$

$0.023^{*}\left(-0.013^{*}\right)$

$-0.067^{*}\left(0.092^{*}\right)$

$-0.075^{*}\left(-0.016^{*}\right)$

$-3.98 \mathrm{e}-3^{*}\left(6.83 \mathrm{e}-3^{*}\right)$

$-0.098^{*}\left(0.111^{*}\right)$

$-4.11 \mathrm{e}-3^{*}\left(4.53 \mathrm{e}-3^{*}\right)$

$-3.47 \mathrm{e}-3^{*}\left(7.41 \mathrm{e}-3^{*}\right)$

$-4.12 \mathrm{e}-3^{*}\left(4.95 \mathrm{e}-3^{*}\right)$

$-0.020^{*}\left(0.072^{*}\right)$

$-4.12 \mathrm{e}-3^{*}\left(4.95 \mathrm{e}-3^{*}\right)$

$-0.122^{*}\left(0.232^{*}\right)$

$-3.96 \mathrm{e}-3^{*}\left(7.50 \mathrm{e}-3^{*}\right)$

$-0.062^{*}\left(0.044^{*}\right)$

$-3.90 \mathrm{e}-3^{*}\left(7.14 \mathrm{e}-3^{*}\right)$

$-0.138^{*}\left(0.161^{*}\right)$

$-4.71 \mathrm{e}-3^{*}\left(8.99 \mathrm{e}-3^{*}\right)$

$-0.071^{*}\left(0.093^{*}\right)$

$-3.96 \mathrm{e}-3^{*}\left(7.71 \mathrm{e}-3^{*}\right)$

$0.087^{*}\left(-0.031^{*}\right)$

$-0.111^{*}\left(0.087^{*}\right)$

$-0.095^{*}\left(-0.014^{*}\right)$

$5.04 \mathrm{e}-3^{*}\left(0.017^{*}\right)$

$-4.02 \mathrm{e}-3^{*}\left(3.89 \mathrm{e}-3^{*}\right)$

$-4.29 \mathrm{e}-3^{*}\left(4.39 \mathrm{e}-3^{*}\right)$

$-1.77 \mathrm{e}-3^{*}\left(1.67 \mathrm{e}-3^{*}\right)$

$-3.15 \mathrm{e}-3^{*}\left(3.10 \mathrm{e}-3^{*}\right)$

$-4.17 \mathrm{e}-3^{*}\left(4.60 \mathrm{e}-3^{*}\right)$

$1.13 \mathrm{e}-3^{*}\left(-3.10 \mathrm{e}-3^{*}\right)$

$1.70 \mathrm{e}-3^{*}\left(-1.90 \mathrm{e}-3^{*}\right)$

$1.69 \mathrm{e}-3^{*}\left(-3.47 \mathrm{e}-3^{*}\right)$

$1.73 \mathrm{e}-3^{*}\left(-2.34 \mathrm{e}-3^{*}\right)$

$204 \mathrm{e}-3^{*}\left(-4.48 \mathrm{e}-3^{*}\right)$

$133 \mathrm{e}-3^{*}\left(-3.14 \mathrm{e}-3^{*}\right)$

$2.32 \mathrm{e}-3^{*}\left(-4.43 \mathrm{e}-3^{*}\right)$

$7.23 \mathrm{e}-4^{*}\left(-2.95 \mathrm{e}-3^{*}\right)$

$1.95 \mathrm{e}-3^{*}\left(-3.53 \mathrm{e}-3^{*}\right)$ $-0.025^{*}\left(0.015^{*}\right)$

Notes. Table 8 reports the $\beta$ - and $\gamma$-coefficient (the latter in parentheses) of the asymmetric regression 4 (Eq. (13)) for the realized range corre

indicators (panel A); and also between futures stock indices and stock indicators (panel B). * denotes significance in a $5 \%$ significance level.
The heteroscedasticity and autocorrelation consistent (Newey-West) standard errors used in the calculation of the corresponding significance

$-9.62 \mathrm{e}-4^{*}\left(1.82 \mathrm{e}-3^{*}\right)$ $-6.09 \mathrm{e}-4^{*}\left(1.92 \mathrm{e}-3^{*}\right)$

$-6.33 \mathrm{e}-4^{*}\left(1.84 \mathrm{e}-3^{*}\right)$

$-6.92 \mathrm{e}-4^{*}\left(2.02 \mathrm{e}-3^{*}\right)$

$-6.19 \mathrm{e}-4^{*}\left(1.92 \mathrm{e}-3^{*}\right)$

$-2.49 \mathrm{e}-3^{*}\left(4.05 \mathrm{e}-3^{*}\right)$

$-1.65 \mathrm{e}-3^{*}\left(1.48 \mathrm{e}-3^{*}\right)$

$-1.33 \mathrm{e}-4^{*}\left(1.78 \mathrm{e}-3^{*}\right)$

$1.17 \mathrm{e}-3^{*}\left(-1.87 \mathrm{e}-3^{*}\right)$

$1.43 \mathrm{e}-3^{*}\left(-1.72 \mathrm{e}-3^{*}\right)$

$1.50 \mathrm{e}-3^{*}\left(-2.10 \mathrm{e}-4^{*}\right)$

$8.04 \mathrm{e}-4^{*}\left(1.65 \mathrm{e}-3^{*}\right)$

$-9.79 \mathrm{e}-4^{*}\left(1.76 \mathrm{e}-3^{*}\right)$

$-2.09 \mathrm{e}-3^{*}\left(1.80 \mathrm{e}-3^{*}\right)$

(1.91e-4* $\left(-8.74 \mathrm{e}-4^{*}\right)$

$-1.96 \mathrm{e}-3^{*}\left(1.18 \mathrm{e}-3^{*}\right)$ 
Table 9A

Realized range correlations - Heterogeneity and Jumps-HAR-RV-J model $\beta_{D}$-coefficient $\& \beta_{W}$-coefficient (in parentheses).

\begin{tabular}{|c|c|c|c|c|c|c|c|}
\hline & TRIN & TICK & TIKI & ADV & DECL & UVOL & DVOL \\
\hline \multicolumn{8}{|c|}{ Panel A. Spot stock indices - indicators } \\
\hline INDU & $-7.16 \mathrm{e}-3\left(0.012^{*}\right)$ & $-0.078^{*}\left(-0.043^{*}\right)$ & $0.017^{*}\left(8.05 e-3^{*}\right)$ & 41.326793* $\left(-14.912^{*}\right)$ & $-0.419^{*}\left(-0.182^{*}\right)$ & $0.025^{*}\left(0.011^{*}\right)$ & $-5.73 \mathrm{e}-3^{*}\left(-8.88 \mathrm{e}-3^{*}\right)$ \\
\hline NDX & $3.16 e-3^{*}\left(7.76 e-3^{*}\right)$ & $-0.061 *(-0.036 *)$ & $0.028 *\left(0.011^{*}\right)$ & $-1074^{*}\left(-105.500^{*}\right)$ & $-0.396 *\left(-0.155^{*}\right)$ & $8.64 \mathrm{e}-3^{*}\left(9.66 \mathrm{e}-3^{*}\right)$ & $3.00 e-3^{*}\left(-5.40 e-3^{*}\right)$ \\
\hline INX & $-9.64 e-3^{*}\left(3.62 e-3^{*}\right)$ & $-0.063^{*}\left(-0.041^{*}\right)$ & $0.022^{*}(0.014 *)$ & $38.135^{*}\left(47.542^{*}\right)$ & $-0.420^{*}\left(-0.191^{*}\right)$ & $0.030^{*}\left(0.015^{*}\right)$ & $-4.31 \mathrm{e}-3^{*}\left(-0.011^{*}\right)$ \\
\hline COMPX & $9.48 \mathrm{e}-4^{*}\left(5.32 \mathrm{e}-3^{*}\right)$ & $-0.059^{*}\left(-0.036^{*}\right)$ & $0.028^{*}\left(0.014^{*}\right)$ & $-1,164^{*}\left(-1,123^{*}\right)$ & $-0.415^{*}\left(-0.157^{*}\right)$ & $0.013^{*}\left(0.010^{*}\right)$ & $4.06 \mathrm{e}-3^{*}\left(-6.01 \mathrm{e}-3^{*}\right)$ \\
\hline RUT & $-0.012^{*}\left(4.38 \mathrm{e}-4^{*}\right)$ & $-0.021^{*}\left(-0.016^{*}\right)$ & $0.016^{*}\left(0.011^{*}\right)$ & $-0.400^{*}\left(-74.012^{*}\right)$ & $-0.364^{*}\left(-0.132^{*}\right)$ & $0.028^{*}\left(8.29 \mathrm{e}-3^{*}\right)$ & $-3.39 \mathrm{e}-3^{*}\left(4.24 \mathrm{e}-3^{*}\right)$ \\
\hline OEX & $-0.012^{*}\left(1.79 \mathrm{e}-3^{*}\right)$ & $-0.068^{*}\left(-0.048^{*}\right)$ & $0.023^{*}\left(0.017^{*}\right)$ & $-1,242^{*}\left(-785.100^{*}\right)$ & $-0.423^{*}\left(-0.198^{*}\right)$ & $0.025^{*}\left(0.014^{*}\right)$ & $-2.34 \mathrm{e}-3^{*}\left(-0.013^{*}\right)$ \\
\hline IDX & $-0.013^{*}\left(2.51 \mathrm{e}-3^{*}\right)$ & $-0.024^{*}\left(-0.018^{*}\right)$ & $-0.017^{*}\left(-0.011^{*}\right)$ & $-37.691 *\left(-76.265^{*}\right)$ & $-0.357^{*}(-0.159 *)$ & $0.042^{*}\left(0.012^{*}\right)$ & $-9.00 \mathrm{e}-3^{*}\left(-5.93 \mathrm{e}-3^{*}\right)$ \\
\hline RUI & $-4.96 \mathrm{e}-3^{*}\left(0.012^{*}\right)$ & $-0.069 *(-0.042 *)$ & $0.032^{*}\left(0.028^{*}\right)$ & $-1,262 *(-882.200 *)$ & $-0.419^{*}\left(-0.157^{*}\right)$ & $0.030 *\left(2.64 \mathrm{e}-3^{*}\right)$ & $-7.19 \mathrm{e}-3^{*}\left(-9.47 \mathrm{e}-3^{*}\right)$ \\
\hline RUA & $5.35 \mathrm{e}-3^{*}\left(0.012^{*}\right)$ & $-0.064^{*}\left(-0.036^{*}\right)$ & $-0.017^{*}\left(-0.023^{*}\right)$ & $48.678^{*}\left(28.126^{*}\right)$ & $-0.397^{*}\left(-0.159^{*}\right)$ & $0.026^{*}\left(0.012^{*}\right)$ & $-7.94 \mathrm{e}-3^{*}\left(-4.55 \mathrm{e}-3^{*}\right)$ \\
\hline \multicolumn{8}{|c|}{ Panel B. Futures stock indices - indicators } \\
\hline ES & $5.46 \mathrm{e}-4^{*}\left(5.10 \mathrm{e}-3^{*}\right)$ & $-0.061^{*}\left(-0.022^{*}\right)$ & $-0.015^{*}\left(-0.015^{*}\right)$ & $815.595^{*}\left(727.248^{*}\right)$ & $-0.446^{*}\left(-0.175^{*}\right)$ & $0.012^{*}\left(0.012^{*}\right)$ & $0.014^{*}\left(-9.44 \mathrm{e}-4^{*}\right)$ \\
\hline $\mathrm{NQ}$ & $-6.03 e-3^{*}\left(5.32 e-3^{*}\right)$ & $-0.045^{*}\left(-0.017^{*}\right)$ & $-6.05 e-3^{*}\left(-0.016^{*}\right)$ & $-112.500^{*}\left(-106.600^{*}\right)$ & $-0.430^{*}\left(-0.173^{*}\right)$ & $7.78 e-3^{*}\left(8.81 e-3^{*}\right)$ & $0.015^{*}\left(1.21 \mathrm{e}-3^{*}\right)$ \\
\hline YM & $-0.014^{*}\left(0.011^{*}\right)$ & $-0.081^{*}\left(-0.024^{*}\right)$ & $0.028^{*}\left(0.018^{*}\right)$ & $-13.423^{*}\left(-402.873^{*}\right)$ & $-0.408^{*}\left(-0.183^{*}\right)$ & $9.62 e-3^{*}\left(5.85 e-3^{*}\right)$ & $0.019 *\left(-3.37 e-3^{*}\right)$ \\
\hline TF & $-3.87 \mathrm{e}-3^{*}\left(5.54 \mathrm{e}-3^{*}\right)$ & $-0.035^{*}\left(-0.022^{*}\right)$ & $-0.050^{*}\left(-0.048^{*}\right)$ & $-302.980^{*}\left(-195.214^{*}\right)$ & $-0.479 *\left(-0.172^{*}\right)$ & $0.0154^{*}\left(1.45 \mathrm{e}-3^{*}\right)$ & $0.022^{*}\left(9.04 \mathrm{e}-3^{*}\right)$ \\
\hline EMD & $-0.022^{*}\left(4.41 \mathrm{e}-4^{*}\right)$ & $-0.021^{*}\left(-0.041^{*}\right)$ & $-0.053^{*}\left(-0.056^{*}\right)$ & $-953.883^{*}\left(-920.881^{*}\right)$ & $-0.489^{*}\left(-0.185^{*}\right)$ & $2.45 \mathrm{e}-3^{*}\left(0.010^{*}\right)$ & $0.023^{*}\left(5.08 \mathrm{e}-3^{*}\right)$ \\
\hline
\end{tabular}

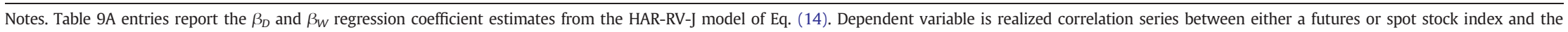

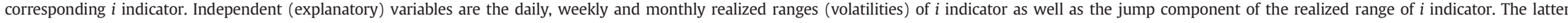
regression coefficient $\left(\beta_{W}\right)$ is in parentheses. ${ }^{*}$ denotes significance in a $5 \%$ significance level.

The heteroscedasticity and autocorrelation consistent (Newey-West) standard errors used in the calculation of the corresponding significance levels; available upon request. 
Table 9B

Realized range correlations - Heterogeneity and Jumps-HAR-RV-J model $-\beta_{M^{-}}$-coefficient $\& \gamma_{\Gamma^{-}}$coefficient (in parentheses).

$$
\text { TRIN }
$$

TICK

TIKI

ADV

DECL

UVOL

DVOL

Panel A. Spot stock indices - indicators

INDU $9.38 \mathrm{e}-3^{*}(0.015)$

NDX $\quad 3.67 \mathrm{e}-3^{*}\left(0.015^{*}\right)$

INX $2.95 \mathrm{e}-3^{*}\left(0.033^{*}\right)$

COMPX 2.62e-3* $\left(0.017^{*}\right)$

RUT $\quad-1.86 \mathrm{e}-3^{*}\left(0.027^{*}\right)$

OEX 7.25e-4* $\left(0.033^{*}\right)$

IDX $\quad-9.63 \mathrm{e}-6^{*}(0.031$

RUI $0.011^{*}\left(0.028^{*}\right)$

RUA $8.03 \mathrm{e}-3^{*}\left(0.018^{*}\right)$

$-0.052^{*}\left(-0.079^{*}\right)$

$-0.046^{*}\left(-0.091^{*}\right)$

TII

ADV

$7.65 \mathrm{e}-3^{*}\left(-0.060^{*}\right)$

$-4.464^{*}\left(114.365^{*}\right)$

$0.023^{*}\left(-0.053^{*}\right)$

59.993* (-99.179*)

$0.017^{*}\left(-0.112^{*}\right)$

$-963.310^{*}\left(1,081^{*}\right)$

$-0.019^{*}\left(-0.134^{*}\right)$

$-78.409^{*}\left(-15.554^{*}\right)$

$-0.043^{*}\left(-0.121^{*}\right)$

$0.020^{*}\left(-0.072^{*}\right)$

$-59.487^{*}\left(73.560^{*}\right)$

$-0.044^{*}\left(0.064^{*}\right)$

$-7.46 \mathrm{e}-3^{*}(0.058)$

$-800.100^{*}\left(1,726^{*}\right)$

$-0.015^{*}\left(0.2179^{*}\right)$

42.205* (29.718*)

$-0.174^{*}\left(0.800^{*}\right)$

$-0.161^{*}\left(0.822^{*}\right)$

$-0.181^{*}\left(0.807^{*}\right)$

$-0.160^{*}\left(0.872^{*}\right)$

$-0.123^{*}\left(0.721^{*}\right)$

$-0.185^{*}\left(0.793^{*}\right)$

$-0.134^{*}\left(0.683^{*}\right)$

$-0.036^{*}\left(-0.106^{*}\right)$

$-0.227^{*}\left(0.931^{*}\right)$

$-0.149^{*}\left(0.758^{*}\right)$

$-0.033^{*}\left(-0.145^{*}\right)$

$-0.025^{*}\left(-0.156^{*}\right)$

$-7.10 \mathrm{e}-3^{*}\left(0.262^{*}\right)$

$4.37 \mathrm{e}-4^{*}\left(0.216^{*}\right)$

787.033* $\left(-964.176^{*}\right)$

$-97.960 *(135.300)$

$-0.171^{*}\left(0.857^{*}\right)$

$0.032^{*}\left(-0.310^{*}\right)$

$-333.972^{*}\left(62.481^{*}\right)$

$-0.169^{*}\left(0.858^{*}\right)$
$-0.190^{*}\left(0.841^{*}\right)$

$-0.059^{*}(-0.115)$

$-0.042^{*}\left(0.378^{*}\right)$

$-226.508^{*}\left(740.488^{*}\right)$

$-0.215^{*}\left(1.040^{*}\right)$

- 952.527* (874.411*)

$-0.196^{*}\left(1.042^{*}\right)$

$0.012^{*}\left(-0.011^{*}\right)$

$0.014^{*}\left(6.79 \mathrm{e}-3^{*}\right)$

$0.016^{*}\left(-0.012^{*}\right)$

$0.013^{*}\left(2.50 \mathrm{e}-3^{*}\right)$

8.02e-3* $\left(-0.011^{*}\right)$

$0.017^{*}\left(-6.33 \mathrm{e}-3^{*}\right)$

$9.39 \mathrm{e}-3^{*}\left(-0.030^{*}\right)$

9.48e- $3^{*}\left(-0.018^{*}\right)$

$0.012^{*}\left(-0.012^{*}\right)$

$-0.054^{*}\left(-0.066^{*}\right)$

$-0.040^{*}\left(0.418^{*}\right)$

$0.013^{*}\left(8.57 \mathrm{e}-3^{*}\right)$

$0.013^{*}\left(0.014^{*}\right)$

$0.012^{*}\left(5.74 \mathrm{e}-3^{*}\right)$

$-1.90 \mathrm{e}-3^{*}\left(-2.83 \mathrm{e}-3^{*}\right)$

$-3.95 \mathrm{e}-3^{*}\left(0.016^{*}\right)$

$-3.30 \mathrm{e}-3^{*}\left(-0.045^{*}\right)$ $-5.78 \mathrm{e}-4^{*}\left(-0.041^{*}\right)$

$-4.71 \mathrm{e}-3^{*}\left(-0.063^{*}\right)$

$1.89 \mathrm{e}-3^{*}\left(-0.043^{*}\right)$

$9.91 \mathrm{e}-3^{*}\left(-0.047^{*}\right)$

$-5.02 \mathrm{e}-3^{*}\left(-0.064^{*}\right)$

$-2.00 \mathrm{e}-3^{*}\left(-0.040^{*}\right)$

$-0.014^{*}\left(-0.066^{*}\right)$

$-1.73 \mathrm{e}-3^{*}\left(-0.032^{*}\right)$

Notes. Table 9B entries report the $\beta_{\mathrm{M}}$ and $\gamma_{J}$ regression coefficient estimates from the HAR-RV-J model of Eq- (14). Dependent variable is realized correlation series between either a futures or spot stock index and the cor-

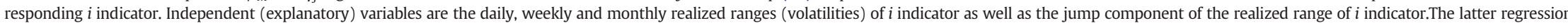
coefficient $\left(\gamma_{J}\right)$ is in parentheses. * denotes significance in a $5 \%$ significance level for either $\beta_{M}$ or $\gamma_{J}$ coefficinet.

The heteroscedasticity and autocorrelation consistent (Newey-West) standard errors used in the calculation of the corresponding significance levels; available upon request. 
Table 10A

Realized range correlations - Heterogeneity and Jumps-HAR-RV-CJ model $-\beta_{c_{D}}$-coefficient and $\beta_{C_{W}}$-coefficient (in parentheses).

\begin{tabular}{|c|c|c|c|c|c|c|c|}
\hline & TRIN & TICK & TIKI & ADV & DECL & UVOL & DVOL \\
\hline \multicolumn{8}{|c|}{ Panel A. Spot stock indices - indicators } \\
\hline INDU & $0.023^{*}\left(0.027^{*}\right)$ & $-0.162^{*}\left(0.220^{*}\right)$ & $-0.042^{*}\left(-0.166^{*}\right)$ & $156.163^{*}\left(205.655^{*}\right)$ & $0.342^{*}\left(0.388^{*}\right)$ & $0.014^{*}\left(6.78 \mathrm{e}-3^{*}\right)$ & $-0.045^{*}\left(-0.046^{*}\right)$ \\
\hline NDX & $0.018^{*}\left(0.026^{*}\right)$ & $-0.155^{*}\left(0.135^{*}\right)$ & $-0.026^{*}\left(-0.121^{*}\right)$ & $-36.090^{*}\left(-1,381^{*}\right)$ & $0.384^{*}\left(0.452^{*}\right)$ & $0.015^{*}\left(3.18 \mathrm{e}-3^{*}\right)$ & $-0.031^{*}\left(-0.051^{*}\right)$ \\
\hline INX & $0.023^{*}\left(0.024^{*}\right)$ & $-0.184^{*}\left(0.154^{*}\right)$ & $-0.091^{*}\left(-0.170^{*}\right)$ & $-60.499^{*}\left(189.151^{*}\right)$ & $0.348^{*}\left(0.354^{*}\right)$ & $0.018^{*}\left(0.013^{*}\right)$ & $-0.059^{*}\left(-0.072^{*}\right)$ \\
\hline COMPX & $0.018^{*}\left(0.026^{*}\right)$ & $-0.147^{*}\left(0.154^{*}\right)$ & $-0.033^{*}\left(-0.111^{*}\right)$ & $-83.580^{*}\left(-1,353^{*}\right)$ & $0.414^{*}\left(0.470^{*}\right)$ & $0.015^{*}\left(4.49 \mathrm{e}-3^{*}\right)$ & $-0.032 *\left(-0.057^{*}\right)$ \\
\hline RUT & $0.014^{*}\left(0.016^{*}\right)$ & $-0.156^{*}\left(0.027^{*}\right)$ & $-0.137^{*}\left(-0.200^{*}\right)$ & $-14.764^{*}\left(288.001^{*}\right)$ & $0.323^{*}\left(0.323^{*}\right)$ & $0.016^{*}\left(0.011^{*}\right)$ & $-0.043^{*}\left(-0.048^{*}\right)$ \\
\hline OEX & $0.021^{*}\left(0.022^{*}\right)$ & $-0.194^{*}\left(0.184^{*}\right)$ & $-0.051^{*}\left(-0.173^{*}\right)$ & $1,078^{*}\left(-371.200^{*}\right)$ & $0.331^{*}\left(0.336^{*}\right)$ & $0.019^{*}\left(0.013^{*}\right)$ & $-0.057^{*}\left(-0.078^{*}\right)$ \\
\hline IDX & $0.018^{*}\left(0.018^{*}\right)$ & $-0.147^{*}\left(0.042^{*}\right)$ & $0.042^{*}\left(0.148^{*}\right)$ & $36.390^{*}\left(78.041^{*}\right)$ & $0.294^{*}\left(0.285^{*}\right)$ & $0.013^{*}\left(7.63 \mathrm{e}-3^{*}\right)$ & $-0.044^{*}\left(-0.051^{*}\right)$ \\
\hline RUI & $0.023^{*}\left(0.027^{*}\right)$ & $-3.55 e-3^{*}\left(-0.090^{*}\right)$ & $-0.224^{*}\left(-0.195^{*}\right)$ & $462.200^{*}\left(1,332^{*}\right)$ & $0.477^{*}\left(0.290^{*}\right)$ & $0.012^{*}\left(6.49 \mathrm{e}-3^{*}\right)$ & $-0.069^{*}\left(-0.046^{*}\right)$ \\
\hline RUA & $0.024^{*}\left(0.029^{*}\right)$ & $-0.173^{*}\left(0.107^{*}\right)$ & $0.206^{*}\left(0.140^{*}\right)$ & $19.350^{*}\left(182.788^{*}\right)$ & $0.323^{*}\left(0.374^{*}\right)$ & $0.013^{*}\left(7.98 \mathrm{e}-3^{*}\right)$ & $-0.035^{*}\left(-0.050^{*}\right)$ \\
\hline \multicolumn{8}{|c|}{ Panel B. Futures stock indices - indicators } \\
\hline ES & $0.012 *(0.015 *)$ & $-0.209^{*}\left(0.159^{*}\right)$ & $0.255^{*}\left(-0.144^{*}\right)$ & $-148.820^{*}\left(705.800^{*}\right)$ & $0.369^{*}\left(0.427^{*}\right)$ & $0.020^{*}\left(0.013^{*}\right)$ & $-0.069^{*}\left(-0.083^{*}\right)$ \\
\hline NQ & $0.012^{*}\left(0.016^{*}\right)$ & $-0.203^{*}\left(0.073^{*}\right)$ & $0.215^{*}\left(-0.179^{*}\right)$ & $23.610^{*}\left(81.980^{*}\right)$ & $0.384^{*}\left(0.445^{*}\right)$ & $0.021^{*}\left(0.010^{*}\right)$ & $-0.064^{*}\left(-0.085^{*}\right)$ \\
\hline YM & $0.024^{*}\left(0.022^{*}\right)$ & $0.036^{*}\left(-0.113^{*}\right)$ & $-0.286^{*}\left(-0.090^{*}\right)$ & $49.820^{*}\left(-115.700^{*}\right)$ & $0.400^{*}\left(0.280^{*}\right)$ & $0.015^{*}\left(0.011^{*}\right)$ & $-0.095^{*}\left(-0.076^{*}\right)$ \\
\hline TF & $0.013^{*}\left(0.016^{*}\right)$ & $-0.030^{*}\left(-0.385^{*}\right)$ & $0.336^{*}\left(0.418^{*}\right)$ & $438.400^{*}\left(-41.800^{*}\right)$ & $0.518^{*}\left(0.411^{*}\right)$ & $0.012^{*}\left(0.012^{*}\right)$ & $-0.084^{*}\left(-0.081^{*}\right)$ \\
\hline EMD & $9.59 \mathrm{e}-3^{*}\left(9.14 \mathrm{e}-3^{*}\right)$ & $-0.081^{*}\left(-0.277^{*}\right)$ & $0.374^{*}\left(0.478^{*}\right)$ & $-78.160^{*}\left(-916.900^{*}\right)$ & $0.511^{*}\left(0.379^{*}\right)$ & $0.018^{*}\left(0.020^{*}\right)$ & $-0.086^{*}\left(-0.105^{*}\right)$ \\
\hline
\end{tabular}

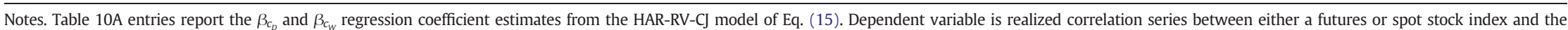

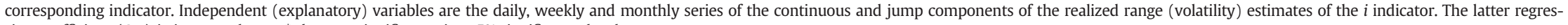
sion coefficient $\left(\beta_{c_{w}}\right)$ is in parentheses. * denotes significance in a $5 \%$ significance level.

The heteroscedasticity and autocorrelation consistent (Newey-West) standard errors used in the calculation of the corresponding significance levels; available upon request. 
Table 10B

Realized range correlations - Heterogeneity and Jumps-HAR-RV-CJ model $-\beta_{c_{M}{ }^{-}}$and $\gamma_{j_{D}}$-coefficient (in parentheses).

$\begin{array}{llll}\text { TRIN TICK } & \text { TIKI }\end{array}$

ADV DECL

UVOL

DVOL

Panel A. Spot stock indices - indicators

INDU $\quad 7.26 \mathrm{e}-3(8.11 \mathrm{e}-3)$

NDX $2.50 \mathrm{e}-3^{*}\left(-4.38 \mathrm{e}-3^{*}\right)$

$0.126^{*}\left(-0.079^{*}\right)$

TIKI

DECL

$-84.449^{*}\left(43.998^{*}\right)$

$0.329 *(-0.367 *)$

$-0.048^{*}\left(0.029^{*}\right)$

$586.034^{*}\left(-1,075^{*}\right)$

$3.31 \mathrm{e}-3^{*}\left(-8.11 \mathrm{e}-3^{*}\right)$

$0.075^{*}\left(-0.061^{*}\right)$

$-0.072^{*}\left(0.024^{*}\right)$

$-94.009^{*}\left(-42.698^{*}\right)$

2.61e-3* $\left(2.48 \mathrm{e}-3^{*}\right)$

$0.074^{*}\left(-0.059^{*}\right)$

$-0.047^{*}\left(0.028^{*}\right)$

$-660.550^{*}\left(-1,162^{*}\right)$

$0.421^{*}\left(-0.339^{*}\right)$

$0.016^{*}\left(0.025^{*}\right)$

$0.024^{*}\left(8.58 \mathrm{e}-3^{*}\right)$

$0.379^{*}\left(-0.368^{*}\right)$

$0.023^{*}\left(0.030^{*}\right)$

$-0.056^{*}\left(-8.13 \mathrm{e}-3^{*}\right)$

$-4.76 \mathrm{e}-3^{*}\left(-0.021^{*}\right)$

$-0.147^{*}\left(0.017^{*}\right)$

$-87.794^{*}\left(8.604^{*}\right)$

$0.023^{*}\left(0.013^{*}\right)$

$0.057^{*}\left(2.61 \mathrm{e}-4^{*}\right)$

$\begin{array}{ll}\text { RUT } & -6.19 \mathrm{e}-3^{*}(-0.012 \\ \text { OEX } & 1.61 \mathrm{e}-3^{*}\left(-9.96 \mathrm{e}-3^{*}\right)\end{array}$

IDX $2.76 \mathrm{e}-3^{*}\left(-0.012^{*}\right)$

$0.105^{*}\left(-0.068^{*}\right)$

$2.76 \mathrm{e}-3^{*}\left(-0.012^{*}\right)$
$0.017^{*}\left(-3.37 \mathrm{e}-3^{*}\right)$

$-0.063^{*}\left(0.024^{*}\right)$

$60.210^{*}\left(-1.204^{*}\right)$

$0.036^{*}\left(-0.024^{*}\right)$

RUA $6.62 \mathrm{e}-3^{*}\left(-6.44 \mathrm{e}-3^{*}\right)$

$0.050^{*}\left(-0.018^{*}\right)$

$0.378 *(-0.319 *)$

$0.023^{*}\left(0.013^{*}\right)$

$-0.060^{*}\left(1.05 \mathrm{e}^{-3} \mathrm{3}^{*}\right)$

$-57.860^{*}(-33.701 *)$

$0.019^{*}\left(0.028^{*}\right)$

$-0.196^{*}\left(0.033^{*}\right)$

$-744.300^{*}\left(-1,272^{*}\right)$

$0.378^{*}\left(-0.371^{*}\right)$

$0.268^{*}\left(-0.018^{*}\right)$

$-18.551^{*}\left(50.957^{*}\right)$

$0.014^{*}\left(0.042^{*}\right)$

$-0.051^{*}\left(-6.41 \mathrm{e}-3^{*}\right)$

$0.381^{*}\left(-0.347^{*}\right)$

$0.018^{*}\left(0.026^{*}\right)$

$-0.082^{*}\left(-6.14 \mathrm{e}-3^{*}\right)$

$-0.047^{*}\left(-0.011^{*}\right)$

Panel B. Futures stock indices - indicators

ES $\quad-4.73 \mathrm{e}-4^{*}\left(-8.76 \mathrm{e}-4^{*}\right)$

NQ $\quad-3.61 \mathrm{e}-3^{*}\left(-5.62 \mathrm{e}-3^{*}\right)$

$0.119^{*}\left(-0.061^{*}\right)$

$9.68 \mathrm{e}-3^{*}\left(-0.013^{*}\right)$

$0.074^{*}\left(-0.045^{*}\right)$

$451 \mathrm{e}-4^{*}$

$-0.152^{*}\left(-0.081^{*}\right)$

$-0.387^{*}\left(-0.016^{*}\right)$

$-0.273^{*}\left(-7.40 \mathrm{e}-3^{*}\right)$

$738.000^{*}\left(813.714^{*}\right)$

$8.300^{*}\left(104.727^{*}\right)$

$0.420^{*}\left(-0.390^{*}\right)$

$-428.788^{*}\left(-8.694^{*}\right)$

$0.163^{*}\left(-0.034^{*}\right)$

$-0.152^{*}\left(0.028^{*}\right)$

$-26.460^{*}\left(-293.560^{*}\right)$

$0.459^{*}\left(-0.371^{*}\right)$

$0.313^{*}\left(-0.362^{*}\right)$

$0.028^{*}\left(0.013^{*}\right)$

$0.490 *($

$-335.533^{*}\left(-936.713^{*}\right)$

$0.469^{*}\left(-0.421^{*}\right)$

$0.030^{*}\left(8.58 \mathrm{e}-3^{*}\right)$

$0.025^{*}\left(0.011^{*}\right)$

$-0.033^{*}\left(-0.010^{*}\right)$

\section{EMD} $-6.49 \mathrm{e}-3^{*}\left(-0.022^{*}\right)$

$-0.146^{*}\left(-0.020^{*}\right)$

$0.441^{*}\left(-0.433^{*}\right)$

$0.020^{*}\left(0.017^{*}\right)$

$-0.076^{*}\left(-9.28 \mathrm{e}-3^{*}\right)$

$-0.088^{*}\left(-0.010^{*}\right)$

$-0.082^{*}\left(-0.014^{*}\right)$

$0.032 *\left(4.69 \mathrm{e}-3^{*}\right)$

$-0.090^{*}\left(0.017^{*}\right)$

10B entries report the $\beta_{c}$ and $\gamma_{j,}$ regression coefficient estimates from the HAR-RV-CJ model of Eq. (15). Dependent variable is realized correlation series between either a futures or spot stock index and the

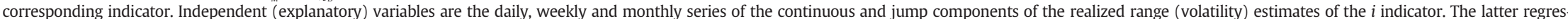
sion coefficient $\left(\gamma_{j}\right)$ is in parentheses. ${ }^{*}$ denotes significance in a $5 \%$ significance level.

The heteroscedasticity and autocorrelation consistent (Newey-West) standard errors used in the calculation of the corresponding significance levels; available upon request. 
Table 10C

Realized range correlations - Heterogeneity and Jumps-HAR-RV-CJ model $-\gamma_{j_{w}}$-coefficient and $\gamma_{j_{M}}$-coefficient (in parentheses).

\begin{tabular}{|c|c|c|c|c|c|c|c|}
\hline & TRIN & TICK & TIKI & ADV & DECL & UVOL & DVOL \\
\hline \multicolumn{8}{|c|}{ Panel A. Spot stock indices - indicators } \\
\hline INDU & $-2.81 \mathrm{e}-3(0.013)$ & $-0.044^{*}\left(-0.053^{*}\right)$ & $6.78 \mathrm{e}-3^{*}\left(8.09 e-3^{*}\right)$ & $-16.355^{*}\left(11.297^{*}\right)$ & $-0.356^{*}\left(-0.322^{*}\right)$ & $0.014^{*}\left(8.21 \mathrm{e}-3^{*}\right)$ & $1.69 e-3^{*}\left(7.93 e-3^{*}\right)$ \\
\hline NDX & $-9.60 e-3^{*}\left(6.37 e-3^{*}\right)$ & $-0.037^{*}\left(-0.047^{*}\right)$ & $0.010^{*}\left(0.023^{*}\right)$ & $-999.300^{*}\left(-914.800^{*}\right)$ & $-0.339 *\left(-0.333^{*}\right)$ & $0.014^{*}\left(7.00 \mathrm{e}-3^{*}\right)$ & $3.64 \mathrm{e}-3^{*}\left(0.011^{*}\right)$ \\
\hline INX & $-0.016 *\left(4.410 \mathrm{e}-3^{*}\right)$ & $-0.042^{*}\left(-0.034^{*}\right)$ & $0.013^{*}\left(0.017^{*}\right)$ & $25.578^{*}\left(56.875^{*}\right)$ & $-0.356^{*}\left(-0.348^{*}\right)$ & $0.017^{*}\left(0.011^{*}\right)$ & $1.27 \mathrm{e}-3^{*}\left(8.64 \mathrm{e}-3^{*}\right)$ \\
\hline COMPX & $-0.015^{*}\left(4.41 \mathrm{e}-3^{*}\right)$ & $-0.036^{*}\left(-0.044^{*}\right)$ & $0.013^{*}\left(0.022^{*}\right)$ & $-1,083^{*}\left(-1,015^{*}\right)$ & $-0.347^{*}\left(-0.340^{*}\right)$ & $0.015^{*}\left(6.02 \mathrm{e}-3^{*}\right)$ & $3.99 \mathrm{e}-3^{*}\left(0.015^{*}\right)$ \\
\hline RUT & $-0.015^{*}\left(3.65 \mathrm{e}-3^{*}\right)$ & $-0.017^{*}\left(-0.019^{*}\right)$ & $9.97 e-3^{*}\left(6.98 \mathrm{e}-3^{*}\right)$ & $-131.449^{*}\left(-71.094^{*}\right)$ & $-0.269^{*}\left(-0.273^{*}\right)$ & $7.12 e-4^{*}\left(6.77 e-4^{*}\right)$ & $0.015^{*}\left(0.023^{*}\right)$ \\
\hline OEX & $-0.018^{*}\left(1.72 \mathrm{e}-3^{*}\right)$ & $-0.048^{*}\left(-0.043^{*}\right)$ & $0.016^{*}\left(0.020^{*}\right)$ & $-837.200^{*}\left(-1,096^{*}\right)$ & $-0.292 *\left(-0.285^{*}\right)$ & $0.015^{*}\left(0.011^{*}\right)$ & $-2.82 \mathrm{e}-4^{*}\left(0.011^{*}\right)$ \\
\hline IDX & $-0.012^{*}\left(-1.23 e-3^{*}\right.$ & $-0.018^{*}\left(-0.013^{*}\right)$ & $-0.010^{*}\left(-7.39 \mathrm{e}-3^{*}\right)$ & $-100.700^{*}\left(-57.261^{*}\right)$ & $-0.359^{*}\left(-0.352^{*}\right)$ & $0.016^{*}\left(5.90 \mathrm{e}-3^{*}\right)$ & $3.05 e-3^{*}\left(7.49 \mathrm{e}-3^{*}\right)$ \\
\hline RUI & $-2.93 e-3^{*}\left(6.34 e-3^{*}\right)$ & $-0.041^{*}\left(-0.044^{*}\right)$ & $0.026^{*}\left(0.018^{*}\right)$ & $-810.312^{*}\left(-816.130^{*}\right)$ & $-0.289^{*}\left(-0.406^{*}\right)$ & $-1.21 \mathrm{e}-4^{*}\left(0.012^{*}\right)$ & $-1.96 \mathrm{e}-3^{*}\left(-8.44 \mathrm{e}-3^{*}\right)$ \\
\hline RUA & $-3.40 \mathrm{e}-3^{*}\left(0.011^{*}\right)$ & $-0.036^{*}\left(-0.036^{*}\right)$ & $-0.022^{*}\left(-0.014^{*}\right)$ & $2.827^{*}\left(54.335^{*}\right)$ & $-0.320^{*}\left(-0.306^{*}\right)$ & $0.015^{*}\left(7.34 \mathrm{e}-3^{*}\right)$ & $4.76 \mathrm{e}-3^{*}\left(4.76 \mathrm{e}-3^{*}\right)$ \\
\hline \multicolumn{8}{|c|}{ Panel B. Futures stock indices - indicators } \\
\hline ES & $-5.13 \mathrm{e}-3^{*}\left(0.011^{*}\right)$ & $-0.022^{*}\left(-0.033^{*}\right)$ & $-0.014^{*}\left(-5.34 \mathrm{e}-3^{*}\right)$ & $-730.000^{*}\left(-794.500^{*}\right)$ & $-0.358^{*}\left(-0.346^{*}\right)$ & $0.012^{*}\left(3.30 \mathrm{e}-3^{*}\right)$ & $0.015^{*}\left(0.024^{*}\right)$ \\
\hline NQ & $-5.77 e-3^{*}\left(6.74 e-3^{*}\right)$ & $-0.017^{*}\left(-0.025^{*}\right)$ & $-0.014^{*}\left(1.54 \mathrm{e}-3^{*}\right)$ & $-135.108^{*}\left(-111.800^{*}\right)$ & $-0.360^{*}\left(-0.356^{*}\right)$ & $8.60 \mathrm{e}-3^{*}\left(2.54 \mathrm{e}-3^{*}\right)$ & $0.018^{*}\left(0.024^{*}\right)$ \\
\hline YM & $-1.77 e-4^{*}\left(-3.13 e-3^{*}\right)$ & $-0.024^{*}\left(-0.059^{*}\right)$ & $0.017^{*}\left(0.031^{*}\right)$ & $-449.622^{*}\left(-314.307^{*}\right)$ & $-0.323^{*}\left(-0.340^{*}\right)$ & $2.95 \mathrm{e}-3^{*}\left(4.14 \mathrm{e}-3^{*}\right)$ & $0.011^{*}\left(0.036^{*}\right)$ \\
\hline TF & $-4.67 \mathrm{e}-3^{*}\left(0.011^{*}\right)$ & $-0.021^{*}\left(-0.048^{*}\right)$ & $-0.045^{*}\left(-0.041^{*}\right)$ & $-216.810^{*}\left(-255.823^{*}\right)$ & $-0.346^{*}\left(-0.420^{*}\right)$ & $-4.68 \mathrm{e}-3^{*}\left(-0.016^{*}\right)$ & $0.027^{*}\left(0.028^{*}\right)$ \\
\hline EMD & $-8.95 \mathrm{e}-3^{*}\left(0.020^{*}\right)$ & $-0.040^{*}\left(-0.054^{*}\right)$ & $-0.053^{*}\left(-0.038^{*}\right)$ & $-913.315^{*}\left(-1,050^{*}\right)$ & $-0.353^{*}\left(-0.386^{*}\right)$ & $5.33 \mathrm{e}-3^{*}\left(-0.027^{*}\right)$ & $0.027^{*}\left(0.037^{*}\right)$ \\
\hline
\end{tabular}

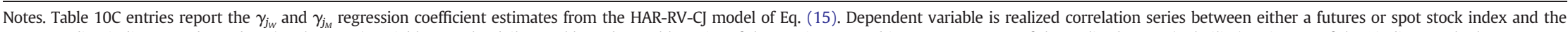

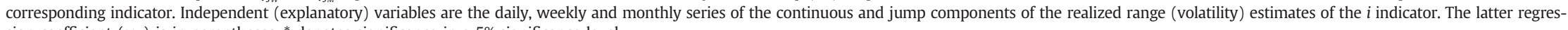
sion coefficient $\left(\gamma_{j_{M}}\right)$ is in parentheses. ${ }^{*}$ denotes significance in a $5 \%$ significance level.

The heteroscedasticity and autocorrelation consistent (Newey-West) standard errors used in the calculation of the corresponding significance levels; available upon request. 


\subsection{Realized correlation estimation}

In the present paper, covariance as well as correlation is estimated non-parametrically. The best non-parametric estimator is the realized volatility estimator in a multivariate level. Barndorff-Nielsen and Shephard (2004) introduced the realized covariance and realized correlation estimator. The realized covariance is given by the cross-products of the two 5-min. Asset returns series throughout each trading day.

$$
R \operatorname{Cov}_{t}^{R V}=\sum_{i=1}^{m / 5} r_{a, i, m, t} \cdot r_{b, i, m, t}
$$

Realized covariance was also discussed in Andersen, Bollerslev, Diebold, and Labys (2001). In the absence of noise, $R C o v_{t}^{R V}$ is a consistent estimator of covariance as the sampling frequency increases. The realized range correlation coefficient $\left(R C_{t}^{R R}\right)$ comes from the $R \operatorname{Cov}_{t}^{R V}$ devided by the square roots of the realized range (volatility) estimates of two assets (RVt,a and $\left.R V_{t, b}^{R R}\right)$.

$$
R C_{t}^{R R}=\frac{R \operatorname{Cov}_{t}^{R V}}{\sqrt{R V_{t, a}^{R R} \sqrt{R V_{t, b}^{R R}}}}=\frac{\sum_{i=1}^{m / 5} r_{a, i, m, t} \cdot r_{b, i, m, t}}{\sqrt{\frac{1}{4 \log (2)} \sum_{i=1}^{m / 5}\left(h_{a, i, m, t}-l_{a, i, m, t}\right)^{2}} \sqrt{\frac{1}{4 \log (2)} \sum_{i=1}^{m / 5}\left(h_{b, i, m, t}-l_{b, i, m, t}\right)^{2}}}
$$

where realized range (volatility) estimates are estimated as in Sub-section 3.1 for each $t$ trading day. Martens and van Dijk (2007) introduced the realized Parkinson range-based volatility estimator-in a univariate level-and Brandt and Diebold (2006) introduced the realized (Parkinson) range-based covariance and correlation estimators.

\subsection{Realized correlation jumps}

The jump detection scheme for correlations employed was introduced and empirically examined in Huang and Tauchen (2005) and Andersen et al. (2007) respectively. As far as the distributional properties of correlation series are not by far different to volatility properties, the test statistic of Andersen et al. (2007) is employed for detecting jumps in realized correlations:

$$
J_{t}^{R C^{R R}}=I\left(\max \left(\left|R C_{t}^{(R R)}-R C_{t}^{(B V)}\right|, 0\right)>c\right) \cdot\left(R C_{t}^{(R R)}-R C_{t}^{(B V)}\right)
$$

where $R C_{t}^{(R R)}$ is the realized range correlation estimator and $R C_{t}^{(B V)}$ is realized bipower-variation correlation estimator. ${ }^{3}$ The threshold $c$ can take different values. The value used throughout the paper is $c=0.05$. The continuous component of correlation is defined as $C_{t}^{R R^{R R}}=R C_{t}^{(R R)}$ - $\max \left(\left|R C_{t}^{(R R)}-R C_{t}^{(B V)}\right|, 0\right)$, where naturally $J_{t}^{R R^{R R}}=\max \left(\left|R C_{t}^{(R R)}-R C_{t}^{(B V)}\right|, 0\right)$. This jump detection test is very strict. So, it captures only high in magnitude jumps.

\section{Preliminary analysis}

The present section of preliminary analysis concentrates on the distributional properties of either volatilities or correlations. For the former, the differences in the distributional properties of volatilities are examined via Kolmogorov-Smirnov test (Subsection 4.1). For the latter, the mean correlations are compared (Sub-section 4.2).

\subsection{Volatilities}

Descriptive statistics do not reveal any incremental information about (spot or futures) stock indices from indicators. ${ }^{4}$ However, the distributional properties' comparison between indicators and the corresponding (spot or futures) indices, via the Kolmogorov-Smirnov (KS) test, reveals this incremental information of indicator. KS test has been widely employed in finance; see, Vassalou and Xing (2004); Lux (2001); Ho, Burridge, Cadle, and Theobald (2000), and Peiró (1999), among others. According

3 The realized bipower variation correlation is

$$
R C_{t}^{(B V)}=\frac{R C v_{t}}{\sqrt{\left.R V_{t, a}^{(B V}\right)} \sqrt{R V_{t, b}^{(B V)}}}=\frac{\sum_{i=1}^{m} r_{a, i, m} r_{b, i, m}}{\sqrt{\mu_{p}^{-2} \sum_{i=2}^{m}\left|r_{a, i, m}\right|\left|r_{a, i-1, m}\right|} \sqrt{\mu_{p}^{-2} \sum_{i=2}^{m}\left|r_{b, i, m}\right|\left|r_{b, i-1, m}\right|}}
$$

where $\mu_{p}=\mathrm{E}\left(|Z|^{p}\right)$ is the mean of the $p$-th absolute moment of a standard normal distribution. For a detailed analysis of the properties of the realized bipower-vatiation estimator, see Barndorff-Nielsen and Shephard (2004).

4 Results are available upon request. 
to Table 1, the indicators' volatility series have different distributional properties to volatility series of the corresponding either spot or futures stock indices.

\subsection{Correlations}

Correlations between the TRIN, ADV, DECL and DVOL indicators and their corresponding either spot or futures stock indices are in-average negative. These indicators reveal incremental information between the corresponding spot and futures stock indices. So, the inclusion of the (spot or futures) indices in a portfolio may result in diversification profits. This is not expected for any stock index as is evident on literature that any spot or futures stock index incorporates the information from the other respective (futures or spot) stock index. The average values of correlations are reported in Table 3. UVOL indicator has the highest, in absolute terms, average values of correlations. The average values of correlations are t-statistically significant. This result may indicate an incremental information in indicators for both spot and futures indices. As evidenced in Andersen, Bollerslev, Diebold, and Ebens (2001), realized (co)variances are extremely right skewed and leptokurtic. In the present paper, this is not evident on realized correlations between indicators and either spot or futures stock indices. Skewness values are close to zero and kurtosis values close to three. So, realized correlations follow an almost normal distribution. Normal distribution is indicated for the TICK-, TRIN- and ADV-correlations via a CVM normality test. ${ }^{5}$

Incremental information of indicators in stock indices may be also indicated via the existence of significant differences in the distributional properties between two correlations. One correlation is between an indicator and the corresponding spot stock index and the second is between the same indicator and the corresponding futures stock index. A Kolmogorov-Smirnov test statistically tests distributional differences. Results are reported in Table 4. The pairs of correlations for all indicators (and their respective indices) have significantly different distributional properties. So, there is incremental information in indicators which affect spot indices differently to the corresponding futures indices.

\section{Asymmetries}

Most of the literature examines asymmetries via granger causality. Granger-causality asymmetries on correlations are analyzed regarding returns- and volatilities-news in detail in Amira et al. (2011). They also perform association tests among returns, volatility and correlations. ${ }^{6}$ The present paper, apart from similar tests in Section 4, examines Granger-causality asymmetries on correlations between indicators and underlying (spot and futures) indices. The scope of asymmetric regressions is to detect, apart from asymmetries, the existence of incremental information from indicators. In order to isolate the explanatory power of asymmetries in volatility (or correlation) series, there is not included any lagged dependent variable as explanatory. However, memory is incorporated via the inclusion of the fractionally integrated volatility (correlation) series as dependent variable instead of just volatility (correlation) series. The estimation method of the asymmetric regression for volatility series is Ordinary Least Squares (OLS).

The error term of the asymmetric regressions is assumed to be iid across all equations for asymmetries (see Amira et al., 2011). Standard errors (S.E.) in all equations are based on the Newey-West estimator of the variance-covariance matrix (see, Dufour, Pelletier, \& Renault, 2006). All regressions can be also performed h-horizons ahead, or by including not lagged by oneperiod independent variables but lagged by h-periods. However, the properties (autocorrelation and memory) of the data make the one lag appropriate for revealing asymmetries. One-period lag is enough and mostly employed in the related literature. The need for an one only lag period is indicated via the distributional properties (serial correlation) of volatility as well as correlation series in the preliminary analysis (Section 4 ) of the present paper. The present section's results are reported in Tables 2, 5-8.

\subsection{Volatilities}

Only one regression is employed for examining asymmetries in volatility series. The asymmetries on volatility of spot or futures stock indices are examined for bad news (negative innovations in returns) and good news (positive innovations in returns) coming from the corresponding indicators. The asymmetric regression for volatility series is:

$$
(1-L)^{d^{R V^{R R}}} R V_{t}^{R R}=\alpha+\beta \cdot D_{+} \cdot r_{t-1}^{i}+\gamma \cdot D_{-} \cdot r_{t-1}^{i}+u_{t}
$$

where $D_{+}=\left\{\begin{array}{ll}1 & r_{t}^{i} \geq 0 \\ 0 & r_{t}^{i}<0\end{array}\right.$ and $D_{-}=1-D_{+}, d^{R V R R}$ is the long-memory estimate of realized range (volatility) estimate, $R V_{t}^{R R}$ is the realized range estimate of either a spot or a futures index and $r_{t}^{i}$ is the returns series of the respective indicator. Results are reported in Table 2. $R^{2}$-values for all volatility series are lower than $2 \%$. This result may be evident because of non-inclusion of a lagged dependent variable as independent. However, asymmetries are significant in both directions for indicators as well as spot and

\footnotetext{
${ }^{5}$ Results are available upon request.

${ }^{6}$ They also checked and confirmed the robustness of their results by: (i) introducing asymmetry in the DCC model as in Cappiello, Engle, and Sheppard (2006), (ii) examining the effect of adding relevant control variables on the asymmetric tests, and (iii) studying the impact of data snooping on the estimation of financial gain after considering asymmetries.
} 
futures stock indices. The asymmetric effect of good news is positive and the one of bad news is negative. They are both as expected.

\subsection{Correlations}

Four regressions are employed for examining asymmetries in correlation series. In order to isolate the explanatory power of asymmetries in correlations, there is not included any lagged dependent variable as explanatory. However, memory is incorporated via the inclusion of the fractionally integrated correlation series as dependent variable instead of just correlation series. The estimation method of all four asymmetric regressions for correlation series is Ordinary Least Squares (OLS). The first two regressions explain correlation via the volatility series of the respective indicator. All the other three regressions model asymmetric effects between correlation and either volatility or returns series of the respective indicator. The second regression concerns an asymmetric effect from indicator's volatility. The third regression models asymmetries via spot or futures returns series demeaned after removing their trend component coming from the respective indicator series. ${ }^{7}$ The last regression directly incorporates the asymmetric effects coming from indicator's good and bad news.

The objective of the first asymmetric regression is to measure the asymmetric effect of the daily volatility of a correlation's $i$ indicator on the correlation between either a spot or futures stock index and the corresponding $i$ indicator. The first asymmetric regression is:

$$
(1-L)^{d_{R C R R}} R C_{t}^{R R}=\alpha+\beta \cdot R V_{t-1}^{i, R R}+u_{t}
$$

where $(1-L)^{d_{R R} R} R C_{t}^{R R}$ is the fractionally integrated realized correlation series as dependent variable to be explained, and $R V_{t}^{i, R R}$ is the realized volatility of the $i$ respective indicator. Results are reported in Table $5 . R^{2}$-values for all correlations are much lower than $1 \%$. This result may be evident because of non-inclusion of a lagged dependent variable as independent. However, asymmetries are significant in both directions. The lagged realized range (volatility) of an indicator significantly explains the correlations between this indicator and the corresponding spot (or futures) indices. All coefficients are significant at a 5\% significance level. Only the TRIN- and ADV-volatilities are negatively related to the correlations between these indicators and their corresponding (spot and futures) stock indices.

Asymmetry in the correlation between (spot or futures) indices and the corresponding indicators is analyzed further in the following three asymmetric regressions. It is examined whether asymmetries come from the realized volatility series of the respective indicator. Realized volatility is decomposed into upturn and downturn volatility, and then their corresponding impact on correlation is examined. So, the second asymmetric regression is:

$$
(1-L)^{d_{R C R R}} R C_{t}^{R R}=\alpha+\beta \cdot D_{+} \cdot R V_{t-1}^{i, R R}+\gamma \cdot D_{-} \cdot R V_{t-1}^{i, R R}+u_{t}
$$

where $D_{+}=\left\{\begin{array}{cc}1 & r_{t}^{i} \geq 0 \\ 0 & r_{t}^{i}<0\end{array}\right.$ and $D_{-}=1-D_{+}$and $R V_{t}^{i, R R}$ are the realized range estimate of the $i$ respective indicator. Coefficients for the second asymmetric regression are presented in Table 6 . Asymmetries are significant in both directions. In good news, indicators' volatility affects positively (as expected) correlations. ${ }^{8}$ In bad news, indicators' volatility negatively affects (as expected) correlations. ${ }^{9}$

Then, it is analyzed the impact of bad news (negative innovations in returns) and good news (positive innovations in returns) on the correlation between (spot or futures) stock indices and the corresponding indicators. The asymmetric impact of the indicator's returns to correlation comes indirectly from the de-meaned returns. The third asymmetric regression is:

$$
(1-L)^{d_{R C R R}} R C_{t}^{R R}=\alpha+\beta \cdot D_{+} \cdot \tilde{r}_{t-1}^{i}+\gamma \cdot D_{-} \cdot \tilde{r}_{t-1}^{i}+u_{t}
$$

where $\widetilde{r}_{t}^{i}=r_{t}-r^{i}, \widetilde{r}_{t}^{i}$ is the de-meaned series of the $r_{t}$ spot or futures return and $\bar{r}^{i}$ is the average of the $i$ indicator returns $\left(r_{t}^{i}\right)$ and the dummy variables are the ones from the second asymmetric regression. This model allows to capture the effect of centered negative and positive returns on correlation through the coefficients $\beta$ and $\gamma$, respectively. A positive sign for $\beta(\gamma)$ means that an increase in the absolute value of the de-meaned return has a positive (negative) effect on future correlation. It also allows us to examine the impacts of large and small negative or/and positive information shocks on correlation. Table 7 presents the coefficients. Asymmetries are significant in both directions. In good news, de-meaned index returns negatively affect (as expected) correlations. $^{10}$ In bad news, de-meaned index returns positively affect (as expected) correlations. ${ }^{11}$

In the last asymmetric regression (asymmetric regression 4), is examined whether there is a significant asymmetric effect directly from the returns of the respective indicator:

$$
(1-L)^{d_{R C R R}} R C_{t}^{R R}=\alpha+\beta \cdot D_{+} \cdot r_{t-1}^{i}+\gamma \cdot D_{-} \cdot r_{t-1}^{i}+u_{t}
$$

\footnotetext{
7 I would like to thank an anonymous referee for his suggestion removing this specific trend from returns.

8 Single exception is ADV- correlations.

9 Exception is the UVOL- correlations.

10 Single exception is UVOL- correlations.

11 Exception is the UVOL- correlations.
} 
where $r_{t-1}^{i}$ is the lagged by 1 period returns for the $i$ respective indicator and the dummy variables are the ones from the fourth asymmetric regression. Table 8 presents the coefficients. Asymmetries are significant in both directions. In good news, indicators' returns affect negatively (as expected) correlations. ${ }^{12}$ In bad news, indicators' returns positively affect (as expected) correlations. $^{13}$

\section{Heterogeneity and jumps}

Solnik, Boucrelle, and Fur (1996) were the first to study, apart from the long-term trends in correlation, the relation between correlation and volatility. Longin and Solnik (2001) used extreme value theory for testing the hypothesis that international equity market correlation increases in volatile times by deriving extreme correlations. More recently, Amira et al. (2011) examined Granger causality between correlations and volatilities via modeling asymmetric behavior.

There was evidence of asymmetries in volatility and correlation series as well, in Section 5. That is why, it is highly expected that heterogeneity in correlation is significant to explain either correlation. Additionally, all volatility and correlation series have high frequency of occurrence of jumps. ${ }^{14}$ That is why, the present section examines the significance as well as explanatory strength of heterogeneity and jumps for correlations. The present section examines: (a) the explanatory power of heterogeneity- and jumps-properties of indicators' volatility in correlations via a heterogeneity- and jumps-model (HAR-RV-J) (Tables 9A and 9B); and (c) the explanatory power of the continuous- and jumps-components of indicators' volatility in correlations via a continuous- and jumps-components model (HAR-RV-CJ) (Tables 10A, 10B, 10C). The family of HAR models is employed for the purposes of this section. HAR models were researched in Corsi (2009) and Corsi, Pirino, and Reno (2010), among others. Significance testing in all section's tables (Tables 9A, 9B and 10A, 10B, 10C) is answered with the use of Newey-West standard errors (S.E.).

The rest of the section examines: (a) the explanatory power of heterogeneity- and jumps-properties of indicators' volatility via HAR-RV-J (Tables 9A, 9B); and (b) the explanatory power of the continuous- and jumps-components of indicators' volatility via HAR-RV-CJ (Tables 10A, 10B, 10C).

Heterogeneity of correlations can also be modeled via the q-period lagged volatilities as well as the volatility-jumps of the $i$ respective indicator. The model employed is symbolized as HAR-RV-J and its equation is indicated as:

$$
R C_{t}^{\mathrm{RR}}=\beta_{0}+\beta_{D} R V_{t-1, t}^{i, R R}+\beta_{W} R V_{t-5, t}^{i, R R}+\beta_{M} R V_{t-20, t}^{i, R R}+\gamma_{J} J_{t-1, t}^{R R^{i, R R}}+\varepsilon_{t}
$$

where $R V_{t}^{i, R R}$ is the realized range (volatility) series of the $i$ indicator, $R V_{t, t+q}^{R R}=q^{-1} \sum_{s=1}^{q} R V_{t+s}^{R R}$ is the mean $q$-period lagged realized range (volatility) and $J_{t}^{R V^{i, R R}}$ is the jump component series of the realized range (volatility) series of $i$ indicator. Dependent variable is realized correlation series between either a futures or spot stock index and the corresponding $i$ indicator. Independent (explanatory) variables are the daily, weekly and monthly realized ranges (volatilities) of $i$ indicator as well as the jump component of the realized range of $i$ indicator. This regression (HAR-RV-J) aims at explaining realized correlations via the significance of heterogeneity and jumps properties of realized volatilities of the $i$ respective indicator. Table 9A presents the $\beta_{D^{-}}$and $\beta_{W^{-}}$-coefficients. Table 9B presents the $\beta_{M^{-}}$and $\gamma_{J}$-coefficients. All coefficients $\left(\beta_{D}, \beta_{W}, \beta_{M}\right.$ and $\left.\gamma_{J}\right)$ are significant. The lagged volatilities in all three frequencies $\left(\beta_{D}, \beta_{W}, \beta_{M}\right)$ negatively explain correlations. Only for the TIKI- and UVOL-correlations, lagged volatilities in all three frequencies $\left(\beta_{D}, \beta_{W}, \beta_{M}\right)$ positively explain correlations. Volatility-jumps negatively explain correlations for the TICK-, TIKI-, UVOL- and DVOL-correlations; for all other correlations, $\gamma_{J}$-coefficients are positive.

Correlations series can be explained via the simultaneous inclusion of the lagged continuous- and jumps-components of the volatility series of the $i$ respective indicator. This model in symbolized as HAR-RV-CJ and structured as:

$$
R C_{t}^{R R}=\left\{\begin{array}{c}
\beta_{0}+\beta_{C_{D}} C_{t-1, t}^{R V^{i, R R}}+\beta_{C_{W}} C_{t-5, t}^{R V^{i, R R}}+\beta_{C_{M}} C_{t-20, t}^{R V^{i, R R}}+ \\
\gamma_{J_{D}} J_{t-1, t}^{R V^{i, R R}}+\gamma_{J_{W}} J_{t-5, t}^{R V^{i, R R}}+\gamma_{J_{M}} J_{t-20, t}^{R V^{i, R R}}+\varepsilon_{t}
\end{array}\right.
$$

where $\beta$-coefficients, in this case, are the coefficients for the lagged continuous components of the $i$ indicator's volatility in daily, weekly and monthly frequencies; and, $\boldsymbol{\gamma}$-coefficients are the coefficients for the lagged jump components of the $i$ indicator's volatility in daily, weekly and monthly frequencies. This regression (HAR-RV-CJ) aims at explaining realized correlations via the simultaneous significance of the continuous and lagged jumps components of realized volatility of the $i$ respective indicator. Table 10A presents the $\beta_{C_{D}}$ and $\beta_{C_{W}}$-coefficients. Table 10B presents the $\beta_{C_{M}}$ and $\gamma_{J_{D}}$-coefficients. Table 10C presents the $\gamma_{J_{W}}{ }^{-}$ and $\gamma_{J M}$-coefficients. All coefficients $\left(\beta_{D C}, \beta_{W C}, \beta_{M C}, \gamma_{D J}, \gamma_{W J}\right.$ and $\left.\gamma_{M J}\right)$ are significant. The continuous-components of volatilities in all three frequencies $\left(\beta_{D C}, \beta_{W C}\right.$ and $\beta_{M C}$ ) negatively explain correlations, only for TIKI- and DVOL-correlations. ${ }^{15}$ The jumps-

\footnotetext{
12 Single exception is UVOL- correlations.

13 Exception is the UVOL- correlations.

14 The frequency of occurrence of jumps in correlations between either a spot or futures stock index and the corresponding $i$ indicator is as high as the frequencies of volatility jumps. In average, frequencies of occurrence of volatility- or correlation- jumps are either close to or even higher than $50 \%$. Volatility- and correlation- jumps series are available upon request.

${ }^{15}$ The coefficients of the daily and monthly continuous components of volatilities $\left(\beta_{C_{D}}, \beta_{C_{M}}\right)$ for TRIN-correlations are negative, as well.
} 
components of volatilities in all three frequencies $\left(\beta_{D C}, \beta_{W C}\right.$ and $\left.\beta_{M C}\right)$ negatively explain correlations, only for TICK-, ADV- and DECL-correlations. ${ }^{16}$

\section{Concluding remarks}

Descriptive statistics do not reveal any incremental information about (spot or futures) stock indices from indicators. However, the fact that indicators' volatility series have different distributional properties to volatility series of the corresponding either spot or futures stock indices via the Kolmogorov-Smirnov (KS) test, reveals the incremental information of indicators for spot and futures corresponding indices.

Negative average correlations between the TRIN, ADV, DECL and DVOL indicators and their corresponding either spot or futures stock indices prove indicators reveal incremental information between the corresponding spot and futures stock indices. Moreover, the statistical significance of the average values of correlations may indicate an incremental information in indicators for both spot and futures indices.

Incremental information of indicators affects spot indices differently to the corresponding futures indices. This is proved via the existence of significant differences (KS test) in the distributional properties between two respective correlations; one correlation is between an indicator and the corresponding spot stock index and the second is between the same indicator and the corresponding futures stock index.

Incremental information is depicted through asymmetric regressions. Indicators' returns significantly explain volatility of the corresponding spot or futures indices in good and bad news as well. Moreover, the lagged realized range (volatility) of an indicator significantly explains the correlations between this indicator and the corresponding spot (or futures) indices. In good (bad) news, indicators' volatility affects positively (negatively), as expected, correlations. In good (bad) news, either de-meaned index returns or indicators' returns negatively (positively) affect, as expected, correlations.

Incremental information of indicators is also proven in the explanatory power of indicators. The significance of heterogeneityand jumps-properties of volatilities in explaining correlations, are evident in either HAR-J or HAR-CJ model. Moreover, the explanatory power of heterogeneity- and jumps-properties of indicators' volatility in correlations via a heterogeneity- and jumps-model (HAR-RV-J) and the explanatory power of the continuous- and jumps-components of indicators' volatility in correlations via a continuous- and jumps-components model (HAR-RV-CJ) are significant.

\section{References}

Amira, K., Taamouti, A., \& Tsafack, G. (2011). What drives international equity correlations? Volatility or market direction? Journal of International Money and Finance, 30(6), 1234-1263.

Andersen, T. G., Bollerslev, T., \& Diebold, F. X. (2007). Roughing it up: including jump components in the measurement, modeling and forecasting of return volatility. Review of Economics and Statistics, 89(4), 701-720.

Andersen, T. G., Bollerslev, T., Diebold, F. X., \& Ebens, H. (2001a). The distribution of realized stock return volatility. Journal of Financial Economics, 61(1), 43-76.

Andersen, T. G., Bollerslev, T., Diebold, F. X., \& Labys, P. (2001b). The distribution of realized exchange rate volatility. Journal of the American Statistical Association, 96(453), 42-55.

Atak, A., \& Kapetanios, G. (2013). A factor approach to realized volatility forecasting in the presence of finite jumps and cross-sectional correlation in pricing errors. Economics Letters, 120, 224-228.

Barndorff-Nielsen, O. E., \& Shephard, N. (2004). Econometric analysis of realized covariation: High-frequency covariance, regression and correlation in financial economics. Econometrica, 72(3), 885-925.

Brandt, M., \& Diebold, F. X. (2006). A no-arbitrage approach to range-based estimation of return covariances and correlations. Journal of Business, 79, 1-13.

Cappiello, L., Engle, R. F., \& Sheppard, K. (2006). Asymmetric dynamics in the correlations of global equity and bond returns. Journal of Financial Econometrics, 4(4), 537-572.

Chen, A., Fung, H. G., \& Kao, E. H. (2008). The dynamic relations among return volatility trading imbalance, and trading volume in the futures markets. Mathematics and Computers in Simulation, 79(3), 429-436.

Chordia, T., Roll, R., \& Subrahmanyman, A. (2011). Recent trends in trading activity and market quality. Journal of Financial Economics, 101(2), $243-263$.

Christensen, K., Oomen, R., \& Podolskij, M. (2010). Realised quantile-based estimation of the integrated variance. Journal of Econometrics, 159(1), 74-98.

Christensen, K., \& Podolskij, M. (2012). Asymptotic theory of range-based multipower variation. Journal of Financial Econometrics, 10(3), 417-456.

Chung, H., Sheu, H., \& Hsu, S. (2010). Trading platform, market volatility and pricing efficiency in the floor-traded and e-mini index futures markets. International Review of Economics and Finance, 19(4), 742-754.

Corsi, F. (2009). A simple approximate long-memory model of realized volatility. Journal of Financial Econometrics, 7(2), 174-196.

Corsi, F., Pirino, D., \& Reno, R. (2010). Threshold bipower variation and the impact of jumps on volatility forecasting. Journal of Econometrics, 159(2), 276-288.

Dufour, J. M., Pelletier, D., \& Renault, E. (2006). Short run and long run causality in time series: inference. Journal of Econometrics, 132(2), 337-362.

Hasbrouck, J. (2003). Intraday price formation in U. S. equity index markets. Journal of Finance, 58(6), 2375-2399.

Hendershott, T., \& Moulton, P. C. (2011). Automation, speed, and stock market quality: The NYSE's hybrid. Journal of Financial Markets, 14, 568-604.

Ho, L. -C., Burridge, P., Cadle, J., \& Theobald, M. (2000). Value-at-risk: Applying the extreme value approach to Asian markets in the recent financial turmoil. PacificBasin Finance Journal, 8(2), 249-275.

Huang X. \& Tauchen, G. (2005). The relative contribution of jumps to total price variance. Journal of Financial Econometrics, 3(4), 456-499.

Liao, Y. (2013). The benefit of modeling jumps in realized volatility for risk prediction: Evidence from Chinese mainland stocks. Pacific-Basin Finance Journal, $23,25-48$.

Longin, F., \& Solnik, B. (2001). Extreme correlation of international equity markets. Journal of Finance, 56(2), 649-676.

Lux, T. (2001). Turbulence in financial markets: The surprising explanatory power of simple cascade models. Quantitative Finance, 1(6), 632-640.

Martens, M., \& van Dijk, D. (2007). Measuring volatility with the realized range. Journal of Econometrics, 138(1), 181-207.

Parkinson, M. (1980). The extreme value method for estimating the variance of the rate of return. Journal of Business, 53, 6-15.

Peiró, A. (1999). Skewness in financial returns. Journal of Banking and Finance, 23(6), 847-862.

Rosa, C. (2011). Words that shake traders. The stock market's reaction to central bank communication in real time. Journal of Finance, 18(5), 915-934.

\footnotetext{
${ }^{16}$ The coefficients of the daily and weekly jumps- components of correlations $\left(\beta_{C_{D}}, \beta_{C_{W}}\right)$ for TRIN- correlations are negative, as well. Moreover, the coefficients of the
} daily jumps- components of correlations $\left(\beta_{C_{D}}\right)$ for DVOL- correlations are also negative. 
Sevi, B. (2013). An empirical analysis of the downside risk-return trade-off at daily frequency. Economic Modelling, 31, $189-197$.

Solnik, B., Boucrelle, C., \& Fur, Y. (1996). International market correlation and volatility. Financial Analysts Journal, 52(5), 17-34.

Soucek, M., \& Todorova, N. (2014). Realized volatility transmission: The role of jumps and leverage effects. Economics Letters, 122, 111-115.

Todorova, N. (2012). Volatility estimators based on daily price ranges versus the realized range. Applied Financial Economics, 22, 215-229.

Todorova, N., \& Soucek, M. (2014). The impact of trading volume, number of trades and overnight returns on forecasting the daily realized range. Economic Modelling, $36,332-340$.

Vassalou, M., \& Xing, Y. (2004). Default risk in equity returns. Journal of Finance, 59(2), 831-868. 